\title{
Eine Krise regionaler Identität und ihr Gebrauchswert für rechtsgerichtete politische Gruppen - ein Beispiel aus Vorpommern
}

\author{
Helmut Klüter \\ Institut für Geographie und Geologie, Universität Greifswald, Greifswald, Deutschland \\ Correspondence: Helmut Klüter (klueter@uni-greifswald.de)
}

Received: 2 July 2019 - Revised: 27 April 2020 - Accepted: 5 May 2020 - Published: 22 June 2020

\begin{abstract}
Kurzfassung. For the first time since the reunification of Germany, right-wing activists and politicians have attempted to take over a university city, i.e. a place where the highly educated, creative, cosmopolitan, innovationoriented groups should be more likely to question irrational populism than elsewhere. An internal organizational problem - in this case: the renaming of the University of Greifswald - which normally should be solved with on-board resources, was shifted to a regional political level as a dispute over Ernst Moritz Arndt. Arndt was one of the most aggressive nationalists in German history, whose name was given to the university under fascist rule in 1933. The dispute was emotionalized by demonstrations and letters to the editor of the regional newspaper, taken up by groups and parties predominantly from the right-wing spectrum. It was brought into a populist form, and pushed with high journalistic effort into the regional public sphere as a Pomeranian identity crisis. In spite of the enormous pressure from outside and the numerous attempts at intimidation, it is admirable that the University Senate members decided to discard the name of Arndt - 63 years after the end of World War II. Although the result of the renaming was noted nationwide, its dramatic circumstances and background were not presented. However, this would have been necessary in order to show how strong right-wing radicalism already is in some regions, by which coalitions it is further enhanced, how strongly it is favoured by the spatial over-centralisation of state institutions, and what a university has to afford in order to assert itself successfully in such an environment.
\end{abstract}

1

Am 18. Januar 2017 beschloss der Senat der Universität Greifswald, die 1933 verordnete Benennung nach Ernst Moritz Arndt zu streichen und zum ursprünglichen Hochschulnamen zurückzukehren. Normalerweise ist die Umbenennung eines Unternehmens, einer Behörde oder einer Körperschaft kein Ereignis, das große Aufmerksamkeit erregt. Doch in diesem Fall folgte eine Protestwelle, die sich in Demonstrationen, einer Flut von Leserbriefen aus ganz Vorpommern an die Regionalzeitung und anderen Aktivitäten äußerte. Grundtenor war, dass Arndt untrennbarer Bestandteil der Identität Vorpommerns sei, dass die Universität dem Rechnung zu tragen habe und daher den Namen Ernst Moritz Arndts beibehalten müsse. Die Vorgänge wurden in der Presse zu einer vorpommerschen Identitätskrise stilisiert und motivierten die erschrockene Landesregierung, den Umbenen- nungsbeschluss aus formalen Gründen zu kassieren. Von einer politisch rechtsorientierten Bürgerinitiative und der AfD wurde dies als Sieg gefeiert. Sie gewann bei der Bundestagswahl am 24. September 2017 im nördlichen Vorpommern mit Greifswald 19,6\% der Stimmen und rückte damit nach der CDU zur zweitstärksten Kraft auf. Die Universität und andere Initiativen bemühten sich in Informationsveranstaltungen und Broschüren um tiefere Erläuterungen zur Rückbenennung. 2018 beschloss der Universitätssenat erneut die Rückkehr zum alten Universitätsnamen, der von der Landesregierung genehmigt wurde. Zwar versuchten rechtsorientierte Kräfte, auch dagegen die Bevölkerung zu mobilisieren. Doch das gelang angesichts der veränderten Informationssituation nur teilweise. Bei den Kommunalwahlen am 26. Mai 2019 musste die AfD in Greifswald Einbußen gegenüber der Bundestagswahl hinnehmen und kam auf 12,3\% der Wählerstim- 
men. Damit wurde sie nach CDU, „Grünen“ und „Linken“ viertstärkste Partei.

Im Folgenden soll erläutert werden, wie es rechtsorientierten Kräften gelang, die Bevölkerung einer Region durch eine Krise zu verunsichern und zumindest teilweise für sich einzunehmen. Damit wird der im sozialwissenschaftlichen Krisendiskurs übliche Makro-Ansatz der globalen, kontinentalen oder nationalen Ebene (vgl. Merkel, 2015) verlassen und durch eine mesoregionale Komponente ergänzt. Anders als bei der Verwendung des gängigen Begriffs „Krisenregion“ als Objektort, an dem sich eine meist umfassendere, gröBere politische Krise manifestiert (vgl. Korf und Schetter, 2012:149) wäre zu fragen, inwieweit neben der Betroffenheit auch die Entstehung einer Krise regional gedacht werden kann. Dabei wird zunächst das Umschlagen von Normalität in eine Krise hinterfragt. Es geht weniger um die Ereignisse und Inhalte, die eine Krise auslösen, sondern ebenfalls um die Organisationen oder Personen, die einen Krisenstatus als solchen feststellen oder ausrufen. In den darauffolgenden beiden Abschnitten werden Erklärungsversuche für die besondere Stärke der politischen Rechten in MecklenburgVorpommern kommentiert und einige besondere Rahmenbedingungen für deren Wachstum aufgezeigt. Dem schließt sich eine kurze Charakteristik der heutigen Bedeutung Ernst Moritz Arndts für Politik und Gesellschaft in der Region an. Auf dieser Basis werden der Verlauf und bisherige Ergebnisse der Rückbenennungskrise dargestellt. Abschließend wird diskutiert, ob das regionale Krisen-Beispiel sich in Vorpommern oder anderswo wiederholen kann.

\section{Normalität und Krise}

Sprachlich oszilliert Krise zwischen einer meist negativ bewerteten Wendung und einem unerwarteten Ereignis, das man auch als Unglück, Schicksalsschlag oder Konflikt bezeichnen könnte, deren räumliche Dimension interessante Anschlussmöglichkeiten für Geographie bietet (vgl. Runkel und Everts, 2017).

Ausgehend von Krise als außergewöhnlichem Schadensereignis oder außergewöhnlicher Schadenserwartung wurde über diesen Begriff eine Metaphorik entwickelt, die fast alle Lebensbereiche erfasst: Wirtschaftskrise, Unternehmenskrise, Demokratie-Krise (Merkel, 2015; Münkler, 2017), Flüchtlingskrise, Brexit-Krise, Finanzkrise, Eurokrise, geopolitische Krise, Vielfachkrise, Fordismuskrise oder legitimatorische Krise (vgl. Oßenbrügge, 2018). Mehr oder weniger offen appelliert der Ausrufer oder Initiator eines Krisendiskurses an die Verlust- oder Existenzängste der Adressaten. Deren Sicherheitsinteressen sollen sie zu denjenigen Aktivitäten motivieren, die der Initiator wünscht. Diese werden in der Regel unter Krisenvermeidung, Prophylaxe oder Stimulierung subsumiert und können sich vom empirischen Schadensereignis weit ins Ideologische entfernen, und zwar besonders dann, wenn das erwartete Schadensereignis noch nicht eingetreten ist. Die Tendenz zur Ideologisierung wird dadurch begünstigt, dass die Frage, was als Symptom, was als Ursache, was als Wesen und welche Ereignisabfolge als Krise interpretiert wird, selten allgemeingültig beantwortet wird.

Der Krisenbegriff setzt als Gegenpol eine Vorstellung über Normalität voraus, also darüber, wie etwas im ,täglichen Leben" richtig funktionieren soll. In der Wissenschaft ist dies einer der Ansatzpunkte für Theorien sozialer Systeme (vgl. Luhmann, 1984; Klüter, 1986). Derartige Normalitätsbezüge können für ein und dasselbe Ereignis unterschiedlich ausfallen: Ein Todesfall kann für eine Familie eine Krise auslösen. Für das Beerdigungsunternehmen bedeutet er eine normale Aufgabe, die wirtschaftlich zu regeln ist. Die einer Krise zugrundeliegende oder vorausgehende Normalität muss also relativ zum Akteur gedacht werden.

Das gilt auch für politische Sachverhalte: Während die Parteien, die gerade die Regierung stellen, bestimmte Sachverhalte als Probleme, Un- oder Sonderfälle, besondere Ereignisse, Herausforderungen oder Aufgaben sehen, neigt die jeweilige Opposition dazu, dieselben Vorgänge als Skandal, Krise oder Komponenten einer sowieso bekannten Krise anzusprechen, die mindestens durch den Rücktritt eines Ministers, besser aber der gesamten Regierung zu bewältigen wäre. Hier dient eine Etikettierung als Krise als Mittel für einen krisenexternen Zweck. Im genannten Beispiel wäre dies die Destabilisierung der Regierung. Wolfgang van den Daeles Frage „Braucht die Gesellschaft ihre Krisen?“” (van den Daele, 2006:35) wurde seinerzeit mit „Ja“ beantwortet.

Die Frage, wie intensiv sich eine Störung entwickelt und wie schnell sie sich ausbreiten kann, muss wiederum relativ beantwortet werden. Die Eskalation eines Problems oder eines Fehlers über Störung, Skandal und andere Dysfunktionalitäten hin zur Krise kann nur in Abläufen geschildert werden, die die Reaktionen von anfeuernden oder rettenden Organisationen und Strukturen berücksichtigen. Deeskalation, Konfliktvermeidung und die Verbesserung von intersystemischer Kompatibilität sind Hauptaufgaben moderner Raumplanung und wichtige Motive für neue Regionalisierungen. Man weist widersprüchlichen gesellschaftlichen Aktivitäten unterschiedliche Grundstücke oder Flächen zu, legt Pufferzonen dazwischen an und schafft durch räumliche Entwicklungspläne langfristige Planungssicherheit für die beteiligten Interessenträger (vgl. Klüter, 2002:150-154).

In die sprachliche und regionale Identifikation von Krisen fließen die in jener Region üblichen Wertungs- und Bewertungsmaßstäbe von sozialen Systemen, ihren Leistungen, Problemen, ihrer Resilienzfaktoren, Umgebungsinformation und die Strategien der Kommunikationsunternehmen aus Internet, Presse, Rundfunk und Fernsehen ein. Mit ihrer Hilfe wird darüber entschieden, ob ein Sachverhalt oder Prozess als „,notwendiges Übel“, als schnell zu korrigierender Fehler oder als Krise eingestuft wird. Auch die Zuordnung einer Krise parallel zur Fragmentierung von Öffentlichkeit in die gesellschaftlichen Teilsysteme Politik, Wirtschaft, Religion, 
Kunst, Familie, Wissenschaft und andere wird trotz technisch möglicher weltgesellschaftlicher Erreichbarkeit angesichts des drastischen Selektionsdrucks nach den jeweiligen national oder regional begrenzten Kommunikationsreichweiten geregelt (vgl. Klüter, 2000b).

Die Suche nach dem richtigen Krisenmanagement und Korrekturstrategien ruft in komplizierten Fällen die Wissenschaft auf den Plan. Korrektur kann auch darin bestehen, dass man sich mit der Existenz bestimmter Krisen abfindet oder anfreundet, sie also als Dauererscheinung ontologisiert. Das wiederum gestattet, dauerhaft Angst zu reproduzieren und die außergewöhnlichen Maßnahmen zur Krisenbewältigung permanent zu installieren. Der ,kalte Krieg“ oder der „Kampf der Kulturen“ (Schmitthenner, 1951; Huntington, 1996) belegen, wie profitabel die Verlängerung eines Krisenzustands für Wissenschaftler sein kann, wenn sie mit imposanten Schreckensbildern die Rechtfertigung für erhöhte Verteidigungs- und Sicherungsausgaben liefern. Manche liefern auch im Voraus: Als Beispiel sei der Hobby-Historiker Peter Sloterdijk genannt, der in seinem Buch „Die schrecklichen Kinder der Neuzeit" Falsches und Stereotypen über Russland in der Manier Merežkovskijs - aber leider nicht mit dessen profundem Wissen - wieder aufwärmt (vgl. Sloterdijk, 2015:144-179). In eindrucksvoller Weise wird hier Wolfgang van den Daelens (2006) Befund bestätigt.

Nicht nur ein kontinental- oder globalpolitischer Rahmen (Luhmann: Weltgesellschaft), auch die Langeweile der Provinz scheint hin und wieder eine aufrüttelnde Krise zu erfordern, sonst kann man keine Zeitung mehr verkaufen. In diesem Kontext ist Hartmut Wewetzers „Kochrezept für einen Skandal" (Wewetzer, 2006:57-58) nach wie vor aktuell. Besonders ergiebig erscheinen in dieser Beziehung Identitätskrisen (vgl. Luhmann, 1997:664-667; 866-1149). Für sie ist Fremdidentifikation nahezu überflüssig, weil sie sich mit Selbstbeschreibung befassen. Auf diese Weise können soziale und psychische Systeme quasi aus sich selbst heraus Krisen erzeugen, ohne dass es dazu größerer existenzgefährdender Anstöße von außen bedarf. Die Anlässe können von den Akteuren knetgummiartig modelliert oder kaugummiartig aufgeblasen werden. Das gilt nicht nur im kleinräumigen, sondern auch im großräumigen Maßstab: Die Selbstbeschreibungsfrage, ob Großbritannien sich der EU zugehörig fühlt, entwickelte sich zur Brexit-Krise, nachdem man sie ohne große Notwendigkeit in einem Plebiszit „dem Volk“ gestellt hatte und dabei die Antwort erhielt, die bestimmte Massenmedien durch jahrelange Propaganda vorbereitet hatten.

Aus diesen Bemerkungen ergeben sich mehrere Arbeitsfragen:

1. Wie funktioniert in einer Region die Abkopplung von nationalen und globalen Kommunikationsströmen, so dass sich ein regionales Identitätsproblem bilden kann?

2. Wie wird ein regionales Problem zur Krise?
3. Inwieweit stützt ein derartiger (Krisen-)Regionalismus konservative und/oder rechtsradikale Tendenzen?

4. Gilt die im bayrischen Kontext aufgedeckte rechtsradikale Strategie „Wir erobern die Städte vom Land aus“ (Becher, 2012:28) auch für Mecklenburg-Vorpommern?

5. Kann die These „Gesellschaftliche Krisenphänomene ermöglichen rechtsextreme Rekrutierung" (Becher, 2012:28) für Mecklenburg-Vorpommern bestätigt werden?

6. Welche Aspekte regionaler Krisen sind dauerhaft wirksam, welche sind verallgemeinerungsfähig?

Am Beispiel des Konflikts um die Rückbenennung der ErnstMoritz-Arndt-Universität in Universität Greifswald - also die Bezeichnung, die sie bis 1933 fast 477 Jahre lang geführt hatte - und der daraus erwachsenen regionalen Krise soll versucht werden, diese Fragen zu beantworten.

\section{Erklärungsansätze für die besondere Stärke der politischen Rechten}

Vor dem Hintergrund der Wahlergebnisse der letzten 5 Jahre scheint der Zusammenhang zwischen den erstarkenden rechtsorientierten Gruppen und der Verteidigung der Benennung der Universität nach dem nationalistischen $\mathrm{Pu}$ blizisten E. M. Arndt trivial zu sein. Leibert and Haunstein (2018) und Leibert (2019) kommen bei der Interpretation der Bundestags- und Europawahlergebnisse zu dem Befund, dass Ostdeutschland vor allem aufgrund der hohen AfD-Gewinne ein eigenes Cluster bildet. Auch bei der Begründung für die dahinter vermuteten Trends greift man $\mathrm{zu}$ vereinfachenden Bildern. Oßenbrügge landet nach einer vergleichenden Durchsicht globaler Humangeographie bei dem Befund einer „regressiven Moderne“ (Oßenbrügge, 2018), bietet ältere Ansätze wie sozialräumliche Polarisierung, Postfordismus als Lösungsrahmen an und staunt über „die Widerstandskraft des territorialen Paradigmas außerhalb der Humangeographie, das nach zwei Jahrzehnten geographischer Dekonstruktionsversuche derzeit einen neuen internationalen Nationalismus mit, völkischem ' Grundton über rechtspopulistische Positionen begründet und rechtfertigt... Die Neuentwicklungen der Kultur-/Sozialgeographie haben wenig Wirksames ergeben, um diesen, roll back ' in den Blick zu nehmen und zu verhindern. Ratlosigkeit beschreibt die Situation wohl treffender" (Oßenbrügge, 2018:316).

Förtner et al. (2019) hinterfragen unter anderem den Erfolg der AfD im Landkreis Vorpommern-Greifswald. Er gerät dabei zu einer Region „umfassender Peripherisierung“, wobei er einen „Verlust an Zentralität“ erfahre, der schließlich einen „Raumergreifungsprozess“ durch Rechtsextreme ermögliche (Förtner et al., 2019:36). Die Rückbenennungskrise oder andere für die Exposition des Rechtsradikalismus wichtige Prozesse oder Ereignisse finden bei Förtner et al. (2019) keine Erwähnung. 
Die Annahme einer „umfassenden Peripherisierung“ erscheint zumindest für die vorpommerschen Hauptstädte Greifswald und Stralsund (UNESCO-Welterbe) wenig überzeugend. Auch Usedom und Rügen verlieren - nicht zuletzt durch den anwachsenden internationalen Tourismus und seine direkten Folgen - ihre in den 1990er-Jahren noch spürbare Provinzialität.

„Der Urbanisierungsprozess bringt dort eine Situation hervor, in der sich der ,Widerstand der Betroffenen ' (Lefebvre, 1975a:123, vgl. Kap. 3.3) in alltäglichen Strukturen eines ruralen sozialen Raumes manifestiert. Durch soziale Praktiken rechter Gruppierungen produziert, verhindert er die Herausbildung der Differenz und verankert das Rurale immer weiter im Alltag. Mit den hohen Stimmanteilen der AfD und ihrem Status als ,Volkspartei' zeigen sich rurale Verstetigungstendenzen, weshalb von einer umfassenden Peripherisierung gesprochen werden kann“" (Förtner et al., 2019:37). Dies ist mit der Tatsache, dass die AfD weniger in agrarisch geprägten vorpommerschen Landgemeinden, sondern in einigen hochverstädterten Tourismuszentren auf Usedom zur stimmenstärksten Partei aufgestiegen ist (vgl. Klüter, 2019: 39-41), kaum in Einklang zu bringen.

Dem stellt dieser Beitrag den Vorschlag entgegen, den Stadt-Land-Gegensatz aufzuheben in der Herausbildung des Ruralen im Prozess der Urbanisierung, um dessen unterschiedliche Ausprägungen als Erklärung für die Wahlerfolge der AfD heranzuziehen (Förtner et al., 2019:40).

In diesen Aufsätzen wird offenbar versucht, die besondere Problematik ländlicher Räume mit invertierten Ansätzen aus der Stadtgeographie zu ergründen. Man ahnt, was eine Stadt, ein Zentrum oder Urbanisierung bedeutet, und versucht, von dort aus die ländliche Exotik, Provinzialität oder Barbarei zu erschließen. Implizit wird dabei dem ländlichen Raum ein wie auch immer gearteter theoretischer Eigenwert abgesprochen. Vor dem Hintergrund städtisch geprägter Theoretiker wie Adorno, Lefebrve und Harvey ist der holprige Umweg über das Städtische zum Ländlichen als „Herausbildung des Ruralen im Prozess der Urbanisierung“ vielleicht verständlich. Doch sozialistische und sozialdemokratische Theorie bietet mit William Morris, Natalie und Wilhelm Liebknecht, Leopold Kohr und anderen auch direkte, umwegfreie $\mathrm{Zu}$ gänge zu sozialen Systemen in ländlichen Räumen (Morris, 1890; Kohr, 1995), die den Verhältnissen in Vorpommern eher angemessen wären (vgl. Klüter, 2016:17, 393-410).

Korrekturbedürftig erscheint das immer wieder durchscheinende Stereotyp vom „blauen Osten“. Betrachtet man die Absolutzahlen des Ergebnisses der Bundestagswahl von 2017, relativiert sich dieses Bild.

Die Wählerschaft der AfD ist in Nordrhein-Westfalen fünfmal stärker als in Mecklenburg-Vorpommern (vgl. Abb. 1). Mit 1,9 Millionen kommt Ostdeutschland auf ein knappes Drittel aller AfD-Wählerstimmen.
Die absolute Stimmenzahl hat nicht zuletzt über die Wahlkampferstattung erhebliche Auswirkungen auf die Finanz- und Organisationskraft der Landesverbände. In Mecklenburg-Vorpommern hatte die AfD Ende 2019 nur 845 von insgesamt 34000 Mitgliedern deutschlandweit. Der größte Landesverband Nordrhein-Westfalen kam 2018 auf 5147, Bayern auf 4946 Mitglieder. Insgesamt entfällt ein Viertel aller AfD-Mitglieder auf Ostdeutschland. Diese Zahlen bedeuten, dass die AfD ihre aufwändigen Wahlkämpfe im Nordosten mit finanzieller und personeller Unterstuitzung aus Westdeutschland und aus weiteren politisch rechtsgerichteten Strukturen durchführt.

In vielen Analysen des AfD-Erfolgs in MecklenburgVorpommern wird im üblichen Rahmen des Disparitätenvergleichs (Fina et al., 2019) und des Parteienwettbewerbs argumentiert, wobei der AfD ,ihre“ besonderen Themen zugesprochen werden (vgl. Behm, 2015). Diese parteienbezogene oder oft sogar parteiimmanente Sichtweise vernachlässigt, dass es einige strukturbedingte, also externe Faktoren gibt, die die auf politischen Wettbewerb angelegten CDU, SPD, FDP, Linke, Grüne behindern und damit zumindest indirekt die AfD als „Übriggebliebene“ begünstigen.

\section{Politische Rahmenbedingungen in Mecklenburg-Vorpommern}

Jene indirekte Begünstigung der AfD sei am Beispiel der kommunalen Ebene genauer erläutert:

Kommunale Dienstleistungen werden in MecklenburgVorpommern für die 710 amtsangehörigen Gemeinden von insgesamt 750 - auf drei Hierarchie-Ebenen erbracht: Gemeinden, Ämter und Landkreise. Die amtsangehörigen Gemeinden haben zwar laut Kommunalverfassung politische Allzuständigkeit, aber abgesehen von den wenigen geschäftsführenden Gemeinden keine eigene Verwaltung, um diese umzusetzen. Juristisch und administrativ müssen sie sich von den Amtsverwaltungen vertreten lassen, die zwar Gemeindeverbände, aber keine Gebietskörperschaften sind. Anders als in Rheinland-Pfalz fehlen ihnen demokratisch gewählte Strukturen.

Auf der lokalen Ebene ergibt sich somit das Paradox, dass die Gemeinden über demokratische Legitimation ohne Umsetzungsapparat, und dass die Ämter zwar über Spezialisten und Fachleute verfügen, jedoch nur indirekt legitimiert sind. Außerdem sind sie nach Artikel 125 der Kommunalverfassung M-V im territorialen Zuschnitt und ihrer Existenz von der Administration des Bundeslandes abhängig. Die Ämter, die Verwaltungen der Kreise und Ministerien bilden somit die Säulen der ,administrativen Gewalt“ (vgl. Klüter, 2000b, 2009). Damit sind die dauerhaft beschäftigten Fachleute und Spezialisten gemeint, die den nur auf Zeit gewählten „Laien“ in Legislative und Exekutive nominell nachgeordnet, jedoch professionell und strategisch häufig überlegen sind. 


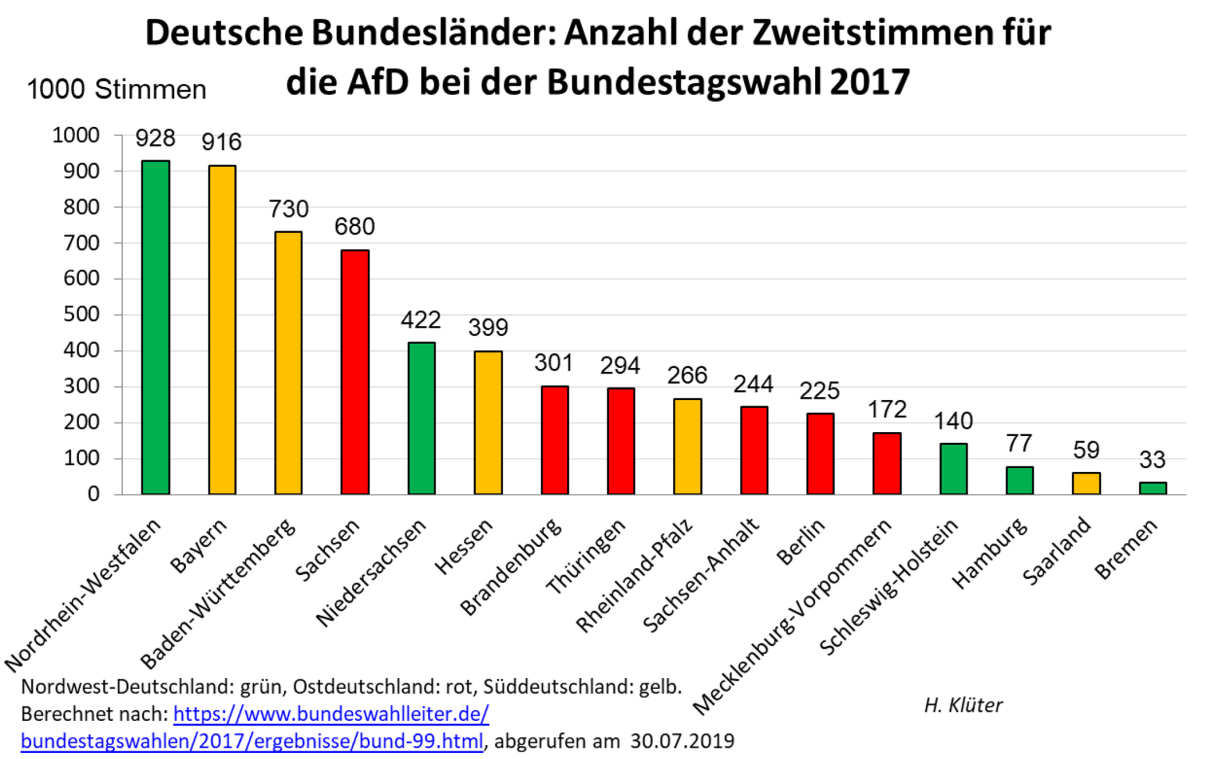

Abb. 1. Deutsche Bundesländer: Anzahl der Zweitstimmen für die AfD bei der Bundestagswahl 2017.

Derzeit zahlen die amtsangehörigen Gemeinden in der östlichen Hälfte Mecklenburg-Vorpommerns $47 \%$ Kreisumlage, $12 \%$ bis $22 \%$ Amtsumlage, $11 \%$ bis $18 \%$ Schulumlage, etwa $20 \%$ Wohnsitzgemeindeanteil für die Kinderbetreuung und $10 \%$ bis $30 \%$ weitere Zweckverbandsumlagen. Über die Hälfte der Gemeinden ist finanziell handlungsunfähig, weil bereits zu Beginn des Haushaltsjahres $80 \%$ bis $140 \%$ der absehbaren Einnahmen verplant sind. Den Bürgermeistern und Gemeinderäten fehlen somit die strategischen Handlungsfreiräume, die für eine politische Profilierung nach demokratischen Parteien nötig wären. Mit anderen Worten: Es ist nahezu irrelevant, welcher Partei der Bürgermeister angehört. Er ist gezwungen, „Nein-Demokratie“ zu praktizieren: Auch die notwendigsten und sinnvollsten Vorschläge von Gemeindemitgliedern müssen unter Hinweis auf die prekäre Finanzlage der Gemeinde abgelehnt werden. Meist bestimmt die Amtsverwaltung - also ein Teil der administrativen Gewalt -, für welche Projekte aussichtsreiche Förderanträge gestellt oder andere Finanzierungsmodelle gefunden werden. Kindergärten und andere soziale Einrichtungen werden veräußert. Nicht selten treten als Käufer Vereine und Strukturen auf, die der politischen Rechten zugerechnet werden. Sie führen die Institutionen in ihrem Sinne weiter. Selbst das Gelände, auf dem jährlich das gegen Rechts gerichtete überregionale Festival ,Jamel rockt den Förster“ stattfindet, wurde von der Gemeinde an eine den Rechten nahestehende Person veräußert.

Die einzige kommunale Ebene, auf der exekutive, legislative und administrative Gewalt in etwa gleichberechtigt nebeneinander standen, war die der Landkreise und kreisfreien Städte. Die östliche Landeshälfte von MecklenburgVorpommern, in der auf $12657 \mathrm{~km}^{2}(=54,3 \%$ der Landesfläche) 719763 Personen (=44,7\% von M-V) wohnen, wur- de bis 1993 von 3 kreisfreien Städten und 17 Landkreisen repräsentiert. Doch genau diese Ebene wurde durch die Kreisgebietsreformen 1994 und 2011 bis zur Funktionsunfähigkeit zentralisiert. Der durchschnittliche Stellenwert einer kreisangehörigen Gemeinde im Landkreis, der 2018 im Saarland bei 0,115 , in Hessen bei 0,050 , in Bayern bei 0,032 und in Deutschland bei 0,027 lag, fiel im Osten MecklenburgVorpommerns von 0,031 im Jahre 1993 auf 0,008 im Jahre 2018. Bereits nach der ersten Reform konnten nicht mehr alle Gemeinden im Kreistag repräsentiert werden. Nach der zweiten Reform 2011 betraf dies in der östlichen Landeshälfte zwei Drittel aller Gemeinden. Damit ist die demokratische Partizipationskette von den Gemeinden zu den Kreisen gebrochen. 2009 wurde der Landtag MecklenburgVorpommern in einer Stellungnahme zur geplanten Kreisgebietsreform gewarnt:

Diese ,Nein-Demokratie“ ist für die Akteure sehr unbefriedigend und für die Bevölkerung desillusionierend. Sie untergräbt ungewollt das positive Image der Demokratie und schafft gezwungenermaßen den Boden für antidemokratische Bewegungen. Je geringer die Kompetenz der Gemeindeoberen, desto höher ist der Wählergewinn für Rechtsextreme (Klüter, 2009:10).

2011 wurden in der östlichen Landeshälfte 3 kreisfreie Städte und 10 Landkreise zu den drei heutigen Großkreisen zusammengelegt. Auf diese Weise wurde die Zahl der jährlichen öffentlichen Rats- und Kreistagssitzungen auf ein Viertel der Zahlen von 2010 reduziert. Unter dem Druck der extrem verknappten Tagungszeit können die Kreistage kaum noch strategisch debattieren, denn sie müssen die Beschlüsse des Landes und die aufgelaufenen Verwaltungsprobleme 


\section{Deutsche Flächenländer: Kreishauptorte auf 10000 km² $^{2}$}

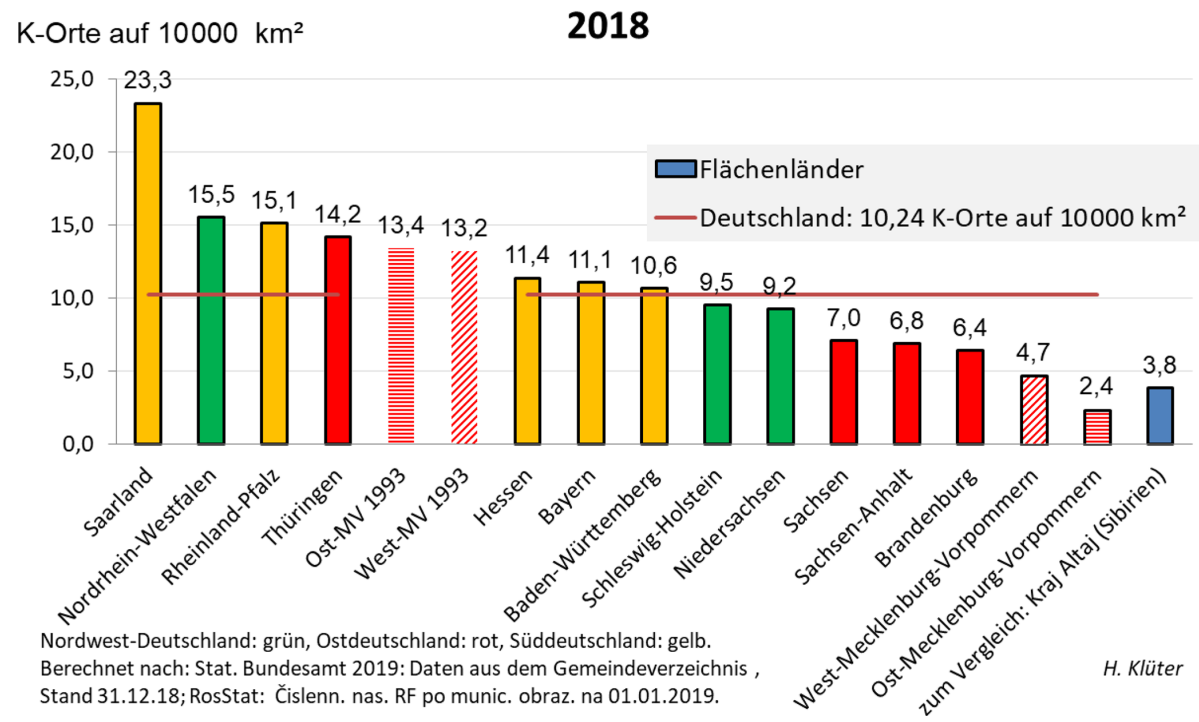

Abb. 2. Deutsche Flächenländer: Kreishauptorte auf $10000 \mathrm{~km}^{2}$ im Jahre 2018.

abarbeiten. Für die Diskussion von investiven Maßnahmen bleiben oft nur wenige Minuten. Die Investitionen auf Kreisebene sind von 2010 bis 2015 auf weniger als die Hälfte geschrumpft und haben sich bis heute nicht erholt. Der politische Spielraum der Kreistage hat sich verringert, der Einfluss der administrativen Gewalt, die die Vorlagen und Basisinformationen erstellt, vergrößert. Kein Kreistagsmitglied ist mehr in der Lage, die lokalen Verhältnisse im gesamten Kreis zu überblicken:

- Der Landkreis Mecklenburgische Seenplatte ist mehr als doppelt so groß wie das kleinste Flächenland Saarland, das immerhin aus 6 Kreisen besteht.

- Die Gemeinde Nadrensee im äußersten Südosten des Landkreises Vorpommern-Greifswald ist von ihrer Kreisstadt $136 \mathrm{~km}$ entfernt, also weiter als von den brandenburgischen Kreisstädten Prenzlau und Eberswalde oder von der Bundeshauptstadt Berlin.

- Die drei verbliebenen Landräte im Osten MecklenburgVorpommerns sind chronisch überlastet und können sich nur noch in Ausnahmefällen um die Politik vor Ort in einer einzelnen Gemeinde kümmern.

- Die Dichte der Kreishauptorte, die 2018 im deutschen Durchschnitt 10,24 auf $10000 \mathrm{~km}^{2}$ betrug, fiel im Osten Mecklenburg-Vorpommerns auf 2,4. Dieser Wert ist für mitteleuropäische Verhältnisse eigentlich unvorstellbar, liegt er doch niedriger als im sibirischen Kraj (Region) Altaj mit 3,8 (vgl. Abb. 2). Der Kraj Altaj ist mit 13,9 Einwohnern je $\mathrm{km}^{2}$ erheblich schwächer als Ost-Mecklenburg-Vorpommern mit 57,9 Einwohnern je $\mathrm{km}^{2}$ besiedelt.
Die Landesadministration hat innerhalb von zwei Jahrzehnten von 17 demokratischen Strukturen - hier: den Kreistagen - 14 aufgehoben. Ähnlich drastisch wurden in ländlichen Räumen Schulen, soziale Einrichtungen und schließlich sogar die Gerichte reduziert. Das alles geschah vor dem Hintergrund in Urbanisierung und Monetarisierung befangener Gutachten, die städtische Pro-Einwohner-Richtwerte rücksichtslos auf den ländlichen Raum übertrugen und nach diesen Maßzahlen Infrastruktureinrichtungen zusammenstrichen. Dabei wurden drastische Verletzungen des Art. 3 GG (Menschenrechte) hingenommen. Mit falschen Bevölkerungsprognosen und abenteuerlich niedrig angesetzten Finanzdaten wurden die Wirtschaftskraft und Steuereinkünfte Mecklenburg-Vorpommerns völlig unterschätzt: Der Landeshaushalt schloss 2019 zum dritten Mal in Folge mit einem Überschuss von über EUR 300 Millionen ab, obwohl die Organisations- und Folgekosten der Kreisgebietsreform die öffentliche Hand immer noch stark belasten. Es dürfte klar sein, dass der Rückzug der Demokratie aus der Fläche geradezu als Einladung an demokratiefeindliche Kräfte verstanden wurde, hier Platz zu greifen. Die Naivität, mit der dies trotz vorheriger Warnungen übersehen wurde, kann man anhand des Abschlussberichts ,Auswirkungen der Landkreisneuordnung" nachvollziehen, mit dem Joachim Jens Hesse, der Chef-Gutachter für die Kreisgebietsreform, sich quasi selbst die Absolution erteilte (Hesse, 2016). Die Begriffe „Rechtsextremismus“ und „Rechtsradikalismus“ kommen bei ihm nicht vor. In dem ausführlichen Abkürzungsverzeichnis und im Text finden alle Parteien Erwähnung, jedoch nicht die AfD. Gleichzeitig mit dem Abschlussbericht erschien Rösels und Sonnenbergs Studie über die Zusammenhänge von Kreisgebietsreform und AfD-Wahlergebnis 


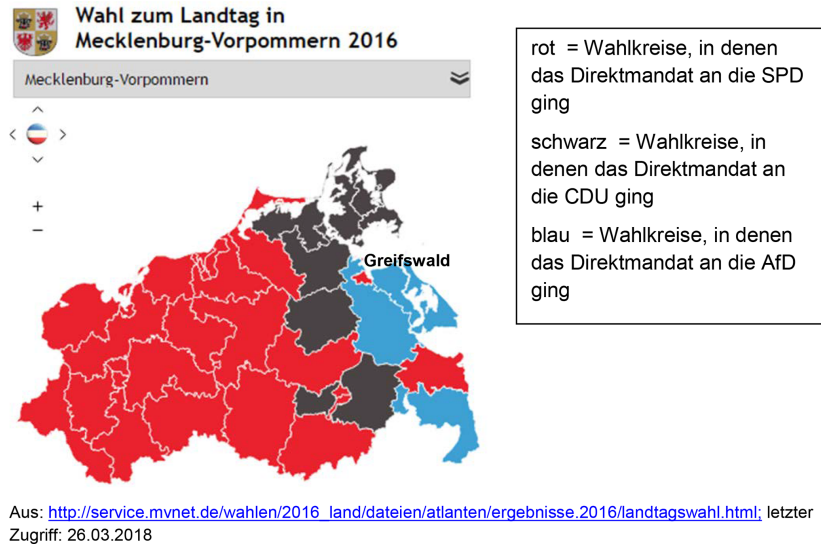

Abb. 3. Wahlkreisergebnisse der Landtagswahl in MecklenburgVorpommern 2016.

(Rösel und Sonnenberg, 2016). Die Studie wurde dem Landtag nicht vorgelegt. Weitere politische Rahmenbedingungen werden von Creuzberger et al. (2018) aufgeführt und diskutiert.

Gründung und Aufstieg der AfD fielen in eine Periode, in der die neu formierten Großkreise mit ihrer organisatorischen Konsolidierung beschäftigt waren - und immer noch sind. Sie können daher nur bedingt ihre demokratischen Funktionen erfüllen. Mecklenburg-Vorpommern ist heute stärker räumlich zentralisiert als zu Zeiten der als überzentralisiert verschrienen DDR. Dieser Kahlschlag hatte auch erhebliche regionalwirtschaftliche Konsequenzen: Mit der Auflösung oder Filialisierung der Kreisverwaltungen verloren 14 Landstädte in Ost-Mecklenburg-Vorpommern jeweils einen ihrer größten Arbeitgeber und Investoren. Damit hat die Landesadministration dort ähnlich destruktiv gewirkt wie die Treuhand-Gesellschaft und die BVVG.

Gespiegelt zu den oben aufgezeigten Problemen, zeigt sich bei den Wahlerfolgen der AfD ein starkes Ost-West-Gefälle (Abb. 3).

Greifswald erscheint als Insel innerhalb des von der AfD dominierten Gebietes. Ein Anlass, auch diese Insel einzunehmen, bot sich unverhofft, als der Senat der Universität Greifswald am 17. Januar 2017 beschloss, den 1933 verordneten Namen „Ernst-Moritz-Arndt-Universität“ abzulegen.

\section{Ernst Moritz Arndt und seine Bedeutung für die politische Rechte in Vorpommern}

Arndt (1769-1860) wurde zwar auf Rügen, also in Vorpommern geboren, kehrte dieser Region jedoch nach seinem Studium und kurzer Beschäftigung an der Universität Greifswald den Rücken. Er verbrachte die meiste Zeit seines Lebens in Bonn, wo er an der dortigen Universität unterrichtete. Er wurde vor allem als Verfasser von weitgehend zeitgebundener Schriften gegen die Leibeigenschaft in Pommern und gegen die französische Vorherrschaft in Europa zur Zeit Na- poleons I. bekannt. Daneben enthält sein Werk jedoch Modernitätsaspekte, die lange Zeit unterschätzt wurden.

In der Periode vor Arndt ging die Aufklärung von einem Menschenbild der einzelnen Person aus, die sich durch Freiheit, Bildung und Chancengleichheit zur Mündigkeit entwickeln sollte. Arndt verwarf den philosophischen Individualismus und ersetzte ihn durch kollektive Identitäten wie beispielsweise das „Volk“, dem der Einzelne sich in Geschichte, Tradition, Kultur, Rasse und Konfrontation mit anderen Völkern unterzuordnen hatte. Die Resignation des Kleinbürgers vor Großstadt, Industrialisierung und staatlicher Bürokratie sowie die damit verbundene Machtlosigkeit erhielten dadurch eine ins Positive gewendete Glorifizierung. Die städtischen Kleinbürger wurden zur zahlenmäßig größten Zielgruppe von Arndts Ideologie. Anders als bei Arndts großen Zeitgenossen Ludwig Uhland und Heinrich Heine sollte das Volk nicht durch ein Parlament, sondern durch eine starke Führungspersönlichkeit repräsentiert werden, mit der Arndt den ,guten Monarchen“ meinte, den jedoch die politische Rechte im Nationalsozialismus schnell für ihre Zwecke umdeutete.

Eine nachhaltige Wirkung auf zukünftige Generationen erzielte Arndt vor allem durch die Lehre während seiner aktiven Hochschullehrerzeit an der Universität Bonn in den letzten beiden Lebensjahrzehnten. In dieser Zeit baute er seinen Franzosenhass zur Erbfeindschaft und einer prinzipiell angelegten Ausländerfeindlichkeit aus. Damit benötigte ein Konflikt oder Krieg keinen konkreten Anlass mehr, sondern Konfliktträchtigkeit ging als Eigenschaft auf das axiomatisch andere ,Volk“ über. Dies rechtfertigte in Preußen die Aufrechterhaltung der allgemeinen Wehrpflicht, die nach den napoleonischen Kriegen in fast allen Staaten wieder abgeschafft worden war. Als Agens für die imaginierte Bedrohung von außen diente der Aufbau von Angst. Sie galt nicht als individualpsychologischer Sachverhalt, sondern als eine Art Kollektivangst, also dieselbe Größe, an die auch bei der Ausrufung einer Krise appelliert wird. Das Volk fungierte - in die heutige Sprache übersetzt - als eine Vereinigung verängstigter Feiglinge. Dies verdeckte Arndt durch entsprechend heldenhafte und kämpferische Gegenbilder aus mystischer Vorzeit. Er verwob Geographisches und Historisches mit teilweise drastischen Vorurteilen zu stark vereinfachten, jedoch einprägsamen Nationalcharakteren, die seiner Meinung nach die ,Völker“ ausmachten. Auf diese Weise wollte er die angebliche Überlegenheit der von ihm idealisierten ,germanischen Rasse“, verkörpert durch einen zukünftigen starken deutschen Staat, beweisen. Diese Überlegenheit setzte eine „Reinheit des Volkes“ voraus, die durch Juden und Ausländer beschädigt werden könnte. Sein letztes größeres Werk „Pro Populo germanico“ (Arndt, 1854), auf Deutsch: „Für/über das deutsche Volk“, besteht aus nur zwei Hauptteilen:

1. „Deutschland und die Deutschen

2. Die Andern“ 
Dieses zweipolige Gliederungsschema (,Wir“ - „die Anderen“) bildet bis heute eines der Grundgerüste populistischer Argumentation. Arndt verknüpfte Halbwissen, Stereotypen und Ressentiments zu einem rassistisch-nationalistischen Gemisch aus kollektiver Angst, diffus gefühlter Krise und latenter Aggression, das er mit der Aura des ,alten Freiheitskämpfers" in der Öffentlichkeit verbreitete.

Trotz dieser überragenden Bedeutung für die Entstehung des modernen Populismus wird Arndt in der Fachwissenschaft, wie etwa Müller (2016), nicht erwähnt.

Instrumentalisiert wurde diese Form des völkischen Nationalismus mit Hilfe eines emotional aufgeladenen Vaterlandsbegriffs, den Arndt in der napoleonischen Zeit maßgeblich mitentwickelt und gegen Frankreich gerichtet hatte. Vaterland war nun die Projektion des Glaubens der Bürger an die Integrität des Staates auf dessen Administrativraum zwecks Erzeugung latenter Aggressivität, der Vaterlandsliebe. Die kollektive Identität, das ,Volk“, erhielt auf diese Weise das „Vaterland“ als Kollektivbesitz und hatte es dementsprechend zu verteidigen. Es wurde als Motivationsrahmen für den straffreien Totschlag im Kriegsfall zum funktionalen Äquivalent des Fürsten: Man tötete oder starb nicht mehr für den König, sondern für das Vaterland (zur Abgrenzung der Raumabstraktion „Vaterland“ von „Administrativraum“ und „Heimat“: Klüter, 2000a:41). Dabei verstärkte der gelernte Theologe Arndt den jeweils aktuellen oder zu erwartenden Kampfauftrag durch eine Verankerung ins Religiöse hinein, der sich die damalige Bevölkerung kaum entziehen konnte, beispielsweise in Arndt (1813). Arndt erwies sich als Meister darin, seine Kriegsverherrlichung in eingängige Gedichte zu verpacken, die die damals kaum alphabetisierte Bevölkerung leicht auswendig lernen konnte.

Einerseits zeigt Arndt mit seiner Idee, eine schnell aufkeimende Industriegesellschaft mit europaweitem Eisenbahnnetz, weltweiten Schifffahrtslinien und exponentiell wachsendem Waren- und Personenverkehr als "Volk“ in ein enges „Vaterland“ mit absoluten territorialen Grenzen zu sperren, wenig Weitblick. Andererseits war ihm klar, dass ein Deutschland mit seiner rassistischen Überheblichkeitsideologie in Europa kaum Freunde finden würde. Es gehört wohl zu den Kuriosa der deutschen Geschichte, dass bereits 1840 das erste Szenario der militärischen Gegnerkonstellation im Ersten Weltkrieg (1914 bis 1918) von dem notorischen Zivilisten Ernst Moritz Arndt entworfen wurde, der - außer zu Duellzwecken - nie eine Waffe getragen hatte. Zu jener Zeit existierte ein vereinigtes Deutschland noch gar nicht. Trotzdem schloss Arndt seine „Erinnerungen aus dem äußeren Leben“" mit 17 Thesen zu einer möglichen deutschen Zukunft $a b$, in denen er mit der geodeterministischen Begründung der territorialen Abrundung eine deutsche Annexion Belgiens und der Niederlande forderte (Arndt, 1842:355-378). Ihm war klar, dass man damit nicht nur die Feindschaft Frankreichs, sondern auch die Großbritanniens heraufbeschworen würde. Im selben Atemzug konstatierte Arndt: „Nach der Lage und Stellung der Völker zueinander ist Russland im Os- ten Deutschlands natürlicher Feind“ (Arndt, 1842:373). Dem damit absehbaren Mehrfrontenkrieg wollte Arndt mit einer Hochrüstungspolitik begegnen, einschließlich des Aufbaus einer mächtigen Kriegsflotte. Er verstand sein Szenario nicht als Warnung, sondern als Herausforderung für neue „Heldentaten".

Arndts Werk wurde schon zu dessen Lebzeiten scharf kritisiert. Bereits 1815 erschien Saul Aschers Schrift „Germanomanie“, in der Arndts antijüdische Tendenz (vgl. Ascher, 1815) und einige seiner - aus heutiger Sicht - rechtspopulistischen Techniken (vgl. Müller, 2016) aufgedeckt wurden. Arndts Zeitgenossen Ludwig Uhland, Joseph von Eichendorff, Philipp Jakob Siebenpfeiffer (1832) und Heinrich Heine huldigten einem friedlichen Nationalismus. Franz Grillparzer prägte 1849 das Epigramm: „Der Weg der neueren Bildung geht von Humanität durch Nationalität zur Bestialität" (Grillparzer, 1960:500). Bereits ein Jahr früher hatte als erster europäischer Flächenstaat die Schweiz eine moderne, multinationale Verfassung bekommen. Nicht zuletzt damit wurde gezeigt, dass Deutsche, Franzosen und Italiener friedlich in einem Staat zusammenleben konnten. Arndts Konzept der Erbfeindschaft und des monarchisch-ständischen Volksstaats als einzigem Zukunftsmodell war schon zu seinen Lebzeiten widerlegt.

Dennoch entfaltete Arndts Werk unter seinen Schülern, zu denen auch der einflussreiche Historiker Heinrich von Treitschke (1834-1896) gehörte, eine erhebliche Breitenwirkung. Der staatliche Administrativraum erfuhr durch die vaterländische Ideologie eine grandiose Aufwertung. Die in Zeiten der Kleinstaaterei eher unbedeutende politische Grenze wurde zur Kultur- und Wirtschaftsgrenze, ihre Veränderung oder Anpassung an Völkisches zum quasi selbstverständlichen Staatsziel erhoben. Arndts in die Zukunft gekehrter Geodeterminismus (Arndt, 1842:355-378) wurde ab 1899 als „Geopolitik“ salonfähig. Militärische Aggression konnte nun mit physiogeographischen, später auch mit wirtschaftsgeographischen „Gegebenheiten“ gerechtfertigt werden, wobei die kolonialistische Expansion als Blaupause oder Testgebiet diente. Geopolitik erwies sich als höchst ergiebiger Krisengenerator, denn ein und dasselbe Gebirge, derselbe Fluss, dieselbe Ebene, dieselbe Küste erhielten aus der jeweils anderen Hauptstadt- oder Regierungsperspektive einen unterschiedlichen ,geopolitischen“"Wert. Daraus konnten dann entsprechend ,unvermeidliche“ Konflikte deduziert werden. Arndts Werke erzielten während der Regierungszeit Wilhelm II. (1888-1918) die höchsten Auflagen ihrer Geschichte, traten ihren Siegeszug durch deutsche Lesebücher an und galten als Rechtfertigung für die Militarisierung der Gesellschaft in Deutschland. Viele Städte, vor allem im damals preußischen Norddeutschland, benannten Straßen, die heute oft in repräsentativen Altbauvierteln liegen, mit dem Namen Arndts.

Nach dem Ersten Weltkrieg ergab sich eine Ernüchterung. Walter Lippmann stellte in seinem 1922 erschienen Buch „Public opinion“ mit den Begriffen „Stereotyp“ und der da- 
durch geschaffenen „Pseudoumwelt“ die analytischen Instrumente zur Verfügung (Lippmann, 1922), mit denen man Arndts Ideen und ihre damalige militaristische Aktualisierung dekonstruieren konnte.

Mit dem Aufkommen des Nationalsozialismus wurde auch der Arndt-Kult wiederbelebt (vgl. Eberle, 2015:6776). Nach der nationalsozialistischen Machtübernahme 1933 wurden in Vorpommern die Greifswalder Universität und 1937 das erst 1929 gegründete Rügensche Heimatmuseum nach ihm umbenannt. Nach dem Zweiten Weltkrieg kam es in Deutschland zu einer kritischen Aufarbeitung der Werke Arndts (vgl. Vogt, 1967; Erhart und Koch, 2007). 2011 wurde die von ihm inspirierte allgemeine Wehrpflicht ausgesetzt. Die Faszination von Arndts Ideologie-Baukasten für die politische Rechte mit den Schlüsselbegriffen Volk, Erbfeindschaft, Überfremdungsgefahr durch Juden- und Ausländer, Bedrohung, Vaterland und immerwährender Gewaltbereitschaft blieb jedoch ungebrochen. Die meisten der Punkte, die hier als Kritik an Arndts Werk geäußert wurden, gelten manchen Rechten mit umgekehrten Vorzeichen als Verdienst (vgl. Metapedia, 2019).

Außerdem gehört Arndt zu den wenigen Säulenheiligen der politischen Rechten, für die eine allgemeine Akzeptanz behauptet wird. Als Beweis dafür galt unter anderem die Tatsache, dass nach Arndt sogar eine Universität benannt wäre - eben die Greifswalder. Nicht zuletzt deswegen wurde die Rückbenennung der Universität von politisch rechtsorientierten Personen und Gruppen so hart bekämpft.

\section{Die Rückbenennung der Universität Greifswald - die Eskalation zur Krise}

In Greifswald ist der Stadthaushalt groß genug, um den politischen Interessenträgern über fiskalisch-administrative Zwänge hinaus strategische Handlungsspielräume zu eröffnen, die die CDU, SPD, Grüne, Linke und FDP für die eigene Profilierung nutzen. Im Gegensatz zum ländlichen Vorpommern stieß die AfD hier auf ein voll funktionsfähiges Parteiensystem. Stärker als im Umland muss sie sich auf Parteienwettbewerb und inhaltliche Diskussionen einlassen.

Greifswald gehörte mit den anderen kreisfreien Städten Rostock, Schwerin, Wismar, Stralsund und Neubrandenburg zu den Orten, die die erste Kreisgebietsreform 1993/1994 unbeschadet überstanden hatten. Die Stadt wuchs von knapp 53000 (2004) auf über 59000 Einwohner im Jahre 2018. Die Universität als mit Abstand größter Arbeitgeber (6243 Beschäftigte, 10019 Studierende, 2019) bestimmt die Stadtentwicklung ähnlich dominant wie die Hochschulen in Freiberg, Marburg oder Tübingen. Rechtsorientierte Gruppen spielten in der Stadtpolitik keine Rolle. Der problemgeschüttelte Mantelkreis Ostvorpommern mit seiner Hauptstadt Anklam und NPD-Umzügen war mental von der Universitätsstadt weit entfernt.
Das änderte sich mit der Kreisgebietsreform 2011. Trotz Protesten aus allen Fraktionen wurde Greifswald die Kreisfreiheit genommen. Die Stadt muss seitdem Kreisumlage zahlen und ist bei vielen strategischen Fragen auf $\mathrm{Zu}$ stimmung und Unterstützung des Landkreises VorpommernGreifswald angewiesen. Andererseits wurde sie Hauptort des Großkreises und erlebt seitdem einen Zuzug von Behörden und Verbänden. Ein besonderer Verband ist der Kreisverband der AfD, der im Herbst 2013 in Heringsdorf gegründet wurde. Zur ersten Vorsitzenden wurde eine Greifswalder Mitarbeiterin der Universitätsverwaltung gewählt, die sich bereits während der Arndt-Debatte 2009/2010 mit ihrer vehementen Ablehnung der Rückbenennung der Hochschule hervorgetan hatte.

Symbolträchtig gestaltete sich die Standortwahl der Geschäftsstelle des Kreisverbands: Er bezog einige Räume in der repräsentativen Villa Am Mühlentor 1. Dort befand sich vorher die Greifswalder SPD-Zentrale, von der aus der spätere Ministerpräsident Erwin Sellering seine Karriere gestartet hatte. Der Kreisverband entwickelte sich schnell zur bedeutendsten legalen rechtsgerichteten Organisation Vorpommerns. Die Durchsetzungskraft der gestärkten Greifswalder AfD zeigte sich bei den Landtagswahlen 2016: Von den 13 AfD-Fraktionsmitgliedern kommen 5 aus Greifswald.

2012 gab sich die Universität Greifswald ein neues Leitbild. Darin heißt es

\section{Die Universität lädt Menschen jeglicher Herkunft und Überzeugung ein, an akademischer Bildung teilzuhaben, gemeinsam für die Erweiterung des Wissens zu arbeiten und Kompetenzen in allen Be- reichen des menschlichen Lebens und Zusammen- lebens auszubilden. Daraus ergibt sich die Ver- pflichtung für sie und für jedes ihrer Mitglieder, in Forschung, Lehre und Studium für eine freiheit- liche, zivile und demokratische Gesellschaft ein- zutreten und sich für das friedliche Zusammenle- ben der Menschen und Völker einzusetzen. Das Be- wusstsein für die eigene Geschichte und die von der Wissenschaft zu bearbeitenden Fragen der $\mathrm{Zu}$ - kunft stellen in ihrer Verbindung eine wesentliche Antriebskraft für die Greifswalder Universität dar (Universität Greifswald, 2012).}

Die kursiv gesetzten Passagen kennzeichnen einige der Gedanken, die mit Arndts Lehren unvereinbar sind.

Vor diesem Hintergrund beschloss der Senat der Universität am 18. Januar 2017 die Rückbenennung der Hochschule, die 1994-1996, 1998-2002, 2008-2011 mehrfach diskutiert worden war. Bereits in der ersten Woche danach wurden zwei Bürgerinitiativen gegründet. Die eine bestand aus stadtbekannten Rechten, die andere aus deren Sympathisanten und weiteren Gegnern der Rückbenennung. Die letztere übernahm unter dem Titel „Ernst Moritz Arndt bleibt“ die Öffentlichkeitsarbeit für die Bewegung (https://de-de.facebook. com/arndt.bleibt/, letzter Zugriff: 23. März 2020). Mit ihrer 
Benennung machte die Initiative deutlich, dass es ihr weniger um die Frage der Universitätsbezeichnung als Voraussetzung eines erfolgreichen Hochschulmarketings als vielmehr um die Zustimmung zu Arndt und seinem Werk ging. Diese Personalisierung des ursprünglichen Anliegens wurde von der Greifswalder Redaktion der „Ostsee-Zeitung“ (OZ) übernommen.

Die Ostsee-Zeitung wurde 1952 als SED-Parteiorgan des damaligen DDR-Bezirks Rostock gegründet. Bereits in den fünfziger Jahren stieg sie zur regionalen Monopolzeitung in ihrem Verbreitungsgebiet auf. Sie wurde 1991 von der Treuhand an die ,Lübecker Nachrichten“ verkauft, bei denen sich 1992 die Axel-Springer-Gruppe und 2009 die MadsackGruppe aus Hannover engagierte. Nach wie vor ist die Ostsee-Zeitung im Gebiet des ehemaligen Bezirks Rostock die einzige bedeutende Tageszeitung.

Die übrigen Landesteile werden ebenfalls von ehemaligen SED-Parteizeitungen mit regionalen Monopolen bedient, und zwar das Gebiet des früheren Bezirks Schwerin von der heutigen ,Schweriner Volkszeitung“ und das des Bezirks Neubrandenburg vom dortigen „Nordkurier“.

Die Greifswalder Lokalredaktion der Ostsee-Zeitung bot den Gegnern der Rückbenennung - auch denen aus der AfD - in den von ihr veröffentlichen Leserbriefen breiten Raum. Am 27. Januar 2017 veröffentlichte sie eine sechsseitige Sonderbeilage unter dem Titel ,Streit um Ernst Moritz Arndt“". Rückbenennungsbefürworter wurden in dieser Diktion zu ,Arndt-Gegnern“, Rückbenennungsgegner zu „ArndtBeschützern“ oder „Arndt-Verteidigern“ erklärt.

In der Greifswalder Ausgabe der Zeitung erschienen von Anfang Januar bis Ende Mai 232 Leserbriefe zum „ArndtStreit“, die von 195 Personen verfasst wurden (Abb. 4). Außerdem erschienen 106 redaktionelle Beiträge dazu, die sich überwiegend gegen die Rückbenennung positionierten. Ein Teil davon wurde auch in den anderen vorpommerschen Ausgaben der Ostsee-Zeitung gedruckt, sodass die Debatte sich über Stralsund und Grimmen bis Rügen sowie in östliche Richtung bis Usedom ausbreitete. In einigen Leserbriefen sah man durch die Rückbenennung die vorpommersche Identität verletzt: „Bei Arndt kommt noch eine tiefe regionale Verwurzelung und Identität dazu“ (OZ vom 24. Januar 2017:10). „Die Universität Greifswald muss weiter den Namen Ernst-Moritz-Arndt-Universität tragen, er stellt auch einen Teil der pommerschen Identität dar" (OZ vom 4. März 2017:12).Von den 191 gegen die Rückbenennung gerichteten Briefen enthielten 118 klassischen Personenkult, nationalistische oder rechtsgerichtete Inhalte, 72 allgemeine oder persönliche Diffamierungen der Rückbenennungsbefürworter. „Heimatlose Wessis“ wollten den alteingesessenen Greifswaldern und Vorpommern ,ihren“ Arndt nehmen. Die Akteure der repräsentativen Demokratie wurden diskreditiert. Pauschal wurde behauptet, zugewanderte Senatsmitglieder wären unqualifiziert, um für die vorpommersche Universität zu entscheiden. Die Ostsee-Zeitung versuchte, ,direkte Demokratie“ umzusetzen. In mehreren Le-

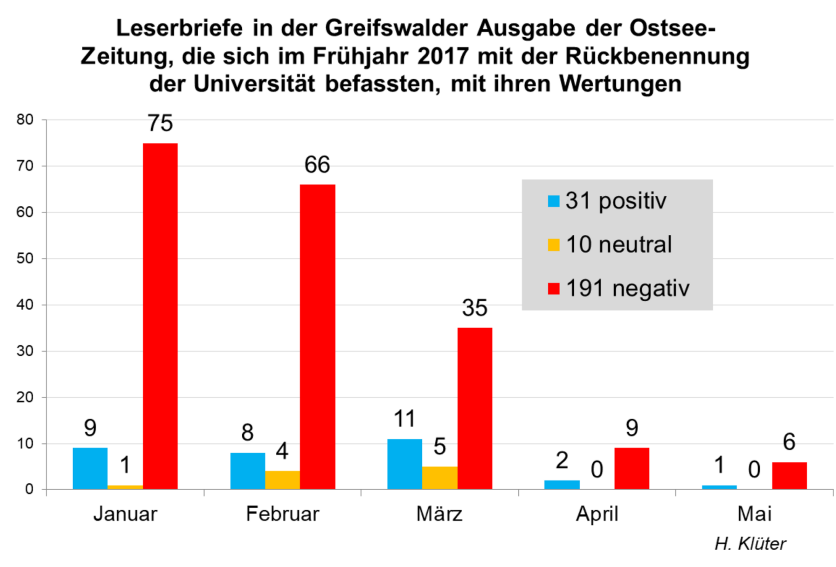

Abb. 4. Anzahl der Leserbriefe in der Greifswalder Ausgabe der Ostsee-Zeitung, die sich im Frühjahr 2017 mit der Rückbenennung der Universität befassten, differenziert nach ihren Wertungen.

serbefragungen wurde über die Rückbenennung und Ernst Moritz Arndt abgestimmt, natürlich immer mit eindeutigen Ergebnissen. Erst im späteren Gespräch gab die Lokalredaktion zu, dass keine wirksamen Kontrollen gegen Mehrfachstimmabgaben eingesetzt wurden. Eine derartige Kampagne hatte es in Vorpommern seit der Wiedervereinigung nicht gegeben (vgl. Baumgartner et al., 2017:5-9).

Die Rückbenennungsbefürworter und Mandatsträger der Universität wurden in der Öffentlichkeit angegriffen, in sozialen Netzwerken beschimpft und persönlich bedroht. Trotz der latenten Gefahr wurden in jenem Zeitraum viele positive Leserbriefe zur Rückbenennung verfasst, von denen die Ostsee-Zeitung 31 veröffentlichte. Eine weitergehende Inhaltsanalyse der veröffentlichten Leserbriefe lieferte Bauer (2018).

Nahezu im wöchentlichen Rhythmus wurden Demonstrationen, Menschenketten und andere Events „,ür Arndt“ organisiert, auf denen Repräsentanten der bürgerlichen Mitte einträchtig neben und hinter denen der politischen Rechten marschierten.

Anfang März 2017 erreichte die Debatte drei Tiefpunkte:

1. Am 4. März kam es auf einer Veranstaltung der Bürgerinitiative ,Ernst Moritz Arndt bleibt“ zur „Prangerrede" des CDU-Fraktionsvorsitzenden der Bürgerschaft auf dem Greifswalder Marktplatz. Dazu hieß es in der Ostsee-Zeitung vom 6. März 2017, 9:

Im Anschluss verlas er die Namen von Bürgerschaftsmitgliedern, die in der namentlichen Abstimmung im Stadtparlament gegen den Antrag seiner Partei votierten, die Universität zum Überdenken des Beschlusses zu bewegen. Jeder Name wurde mit Pfiffen und „Pfui“Rufen vom Publikum quittiert. Im Anschluss bat Hochschild darum, auch Oberbürgermeister Stefan Fassbinder auszupfeifen, da dieser 
die Greifswalder seiner Ansicht nach in puncto Arndt allein lasse.

So etwas hatten die Greifswalder seit der Nazi-Zeit nicht mehr auf ihrem Marktplatz erlebt. In einem Leserbrief derselben Zeitungsausgabe wurde auf S. 5 kommentiert:

... Was sich jedoch am letzten Samstag auf einer Kundgebung ereignete, enttäuscht mich zutiefst: die öffentliche Verlesung von Bürgerschaftsmitgliedern auf dem Marktplatz, die sich in einer ebenfalls öffentlichen Abstimmung nicht der Sache des Kundgebungsredners angeschlossen hatten, und die johlende Menge, die jeden dieser Namen mit Schmährufen quittierte. Alle Menschen mit Anstand, Achtung vor Anderen und Geschichtsbewusstsein sollten ahnen, was es heißt, wenn Namen wie an einem öffentlichen Pranger vor johlender Menge verlesen werden.

2. Einige Rückbenennungsgegner, darunter auch Professoren der Greifswalder rechts- und staatswissenschaftlichen Fakultät, reichten beim Bildungsministerium Rechtsaufsichtsbeschwerden gegen die eigene Universitätsführung ein - mit der angedrohten Konsequenz, im Falle der Nichtbefolgung den Klageweg zu beschreiten. Entgegen der öffentlichen Wahrnehmung handelte es sich dabei bis auf wenige Ausnahmen um Professoren, die aus Westdeutschland berufen oder ernannt worden waren. Bereits 2016 hatte einer von ihnen sich als Abgeordneter für die AfD in den Landtag wählen lassen.

3. Am 7. März 2017 gab das Bildungsministerium dem Druck nach und untersagte der Universität die Rückbenennung aus formalen Gründen. Die AfD triumphierte am selben Tag:

Ernst Moritz Arndt-Universität behält Namen: Ministerin bestätigt AfD-Forderung. Zur Entscheidung des Bildungsministeriums, wonach die „Ernst Moritz Arndt“-Universität ihren Namen wegen Rechtsfehlern im Verfahren nicht ablegen darf, erklärt AfD-Fraktionschef LeifErik Holm: Das ist eine gute Nachricht für alle Greifswalder. Darauf sollten wir heute anstoßen. Der starke öffentliche Druck der Bürger hat diesen Erfolg möglich gemacht. Mit der ministeriellen Entscheidung bestätigt Frau Hesse zudem die Auffassung der AfD-Fraktion, die einen entsprechenden Antrag in die morgige Plenarsitzung eingebracht hat. Offenbar wollte Frau Hesse ihren Genossen ersparen, den fundierten Antrag der AfDFraktion ablehnen zu müssen. Die voreilige
Entscheidung einiger weniger Senatoren, die über die Köpfe der Bürger in einem grenzwertigen Verfahren die Ablegung des Namens durchpeitschten, rächt sich nun bitter. Ohne die Greifswalder Bürger zu berücksichtigen, sollten hier Tatsachen geschaffen werden. Dem wurde nun zunächst ein Riegel vorgeschoben. Klar ist aber, dass die politisch überkorrekten Bilderstürmer nicht aufgeben werden. Die Greifswalder und alle Bürger unseres Landes müssen wachsam bleiben, um weitere Versuche abzuwehren, unsere wichtigen historischen Wurzeln zu kappen. Die Alternative für Deutschland wird diesen Kampf auch weiterhin nach Kräften unterstützen (Holm, 2017).

Bei der Bundestagswahl am 24. September 2017 wurde im Wahlkreis Vorpommern-Rügen - Vorpommern Greifswald I, zu dem auch Greifswald gehörte - die AfD mit 19,6\% der Stimmen zweitstärkste Partei nach der CDU mit $32,9 \%$. Im südlich anschließenden Wahlkreis Mecklenburgische Seenplatte I - Vorpommern-Greifswald II - erzielte sie mit 23,5\% ein noch höheres Ergebnis und rangierte auch dort nach der CDU an zweiter Stelle.

Der Greifswalder Oberbürgermeister Stefan Fassbinder kommentierte jene Krisenzeit in einem OZ-Interview am 12. Januar 2018:11 wie folgt:

Debatten sind positiv ... Was mich beunruhigt, ist die Art und Weise, wie diese Debatte teilweise geführt worden ist. Da sind Worte und Argumente gefallen, die nichts mit der Sache zu tun haben. Wo beispielsweise jemand geografisch herkommt, der eine bestimmte Meinung vertritt, ist nicht relevant. Die Äußerungen waren teilweise sehr verletzend. Ich kenne auch Leute, die sich zu diesem Thema nicht mehr äußern wollen, weil sie Angst haben, angegriffen oder angefeindet zu werden. Das darf nicht mehr passieren, bei keinem Thema. Alle Beteiligten müssen aufpassen, dass es nicht ins Persönliche geht...

\section{Deeskalation der Krise}

Die Prangerrede wurde nicht nur vom Oberbürgermeister als bedrohliches Menetekel empfunden. Manchem wurde der Rückgriff auf nationalsozialistische Hetz-Instrumente erst jetzt klar. Die Ernüchterung darüber ließ die Unterstützung für die Rückbenennungsgegner erheblich schrumpfen.

Da die Ostsee-Zeitung die Position der Rückbenennungsbefürworter extrem verzerrt, verkürzt oder überhaupt nicht wiedergab, entschloss sich eine Arbeitsgruppe aus Mitgliedern der Universität, ihre Argumente in einer eigenen, 45seitigen Broschüre zu veröffentlichen (Baumgartner et al., 2017). Sie erschien am 18. Oktober 2017 unter dem Titel 
„Für die Universität Greifswald“. Damit war das Informationsmonopol der Ostsee-Zeitung für „Papier-Medien“ gebrochen. Die Broschüre trug erheblich zur Versachlichung der Diskussion bei. Das Parallelprodukt der Rückbenennungsgegner erschien zwei Wochen später unter dem Titel „Ja zu Ernst Moritz Arndt. Ja zur Freiheit“". Es umfasst nur 4 Seiten und enthielt keine Artikel von Hochschulangehörigen.

Währenddessen versuchte die Universität, die vom Bildungsministerium am 7. März geäußerten Beanstandungen zu beheben. Parallel dazu wurden im November, Dezember und Januar Meinungsumfragen unter den Mitarbeitern und eine studentische Urabstimmung mit widersprüchlichen Ergebnissen durchgeführt. Die Teilnehmerzahlen an den Demonstrationen der Rückbenennungsgegner gingen drastisch zurück und pendelten sich bei etwa 20 bis 40 Personen ein.

Am 17. Januar 2018 beschloss der Hochschulsenat eine neue Grundordnung, in der ,Universität Greifswald“ als rechtsverbindliche Hochschulbezeichnung festgelegt wurde. Man konzedierte, dass ihr im nichtamtlichen Verkehr von denen, die es wünschten, der Name „Ernst Moritz Arndt“ vorangestellt werden könne. Trotz dieses Kompromisses kam es erneut zu drastischen Kommentaren und erneuten Demonstrationen.

Lorenz Caffier, CDU-Innenminister von MecklenburgVorpommern, postete am selben Tag auf Facebook:

Ich bin enttäuscht über die Entscheidung des Senats der Ernst-Moritz-Arndt-Universität zur Namensänderung. Ich halte diesen Schritt für grundfalsch. Ernst Moritz Arndt war Schriftsteller, ein großer Freiheitskämpfer und setzte sich für ein vereintes Deutschland während der Besetzung durch Napoleon ein. Er ist eine Identifikationsfigur für die gesamte Region und hinterließ ein beachtliches Werk... Die Entscheidung des Universitätssenats ist das Ergebnis von linksgrüner Meinungsmache und einer vollkommen undifferenzierten Diskussion. Ich kann nur hoffen, dass das nicht der Einstieg in eine groß angelegte Namensbereinigungswelle oder gar Kulturrevolution ist (Caffier, 2018).

Mit Caffier beteiligte sich erstmals ein Regierungsmitglied an der Diffamierung der Universität. Unterschiede zur gängigen AfD-Rhetorik (s.o.) sind in diesem Zitat kaum auszumachen. Das SPD-geführte Bildungsministerium genehmigte am 19. April 2018 die neue Grundordnung, und damit auch die Rückbenennung der Universität. Sie trat mit dem 1. Juni 2018 in Kraft. Die Zahl der Protestaktionen und die Beteiligung daran ebbten ab. Die Bürgerinitiative „Ernst Moritz Arndt bleibt" konzentriert sich nun darauf, in Greifswald ein zweites Arndt-Denkmal aufzustellen.

Bereits vorher, im März 2018, hatte Axel Hochschild, der Prangerredner vom 4. März 2017, sein Amt als Vorsitzender des CDU-Stadtverbands abgegeben. Ende 2018 wurde die Leiterin der OZ-Lokalredaktion Greifswald, die die Leserbriefkampagne mit zu verantworten hatte, ausgetauscht. Die neuen Redaktionsmitglieder berichten seitdem erheblich kritischer über politisch rechtsgerichtete Vorkommnisse. Einige Aktivitäten von Rückbenennungsgegnern fanden Eingang in den Verfassungsschutzbericht des Landes (Verfassungsschutz 2018 Mecklenburg-Vorpommern, 2019:70).

\section{Vorläufige Ergebnisse}

Erstmals seit der Wiedervereinigung wurde versucht, von rechts außen eine Universitätsstadt einzunehmen, d.h. einen Ort, in dem die hoch gebildete, bürgerlich-kreative, weltoffene, innovationsorientierte Schicht stärker als in anderen Städten und Regionen vertreten ist. Ein internes Organisationsproblem - hier: die Rückbenennung der Universität Greifswald -, das normalerweise mit Bordmitteln gelöst wird, wurde als Streit um die Person Arndts auf eine regionalpolitische Ebene verschoben. Es wurde von Gruppen und Parteien des politisch rechten Spektrums aufgegriffen, in eine populistische Form (vgl. Müller, 2016) gebracht, mit Hilfe von Demonstrationen und Leserbriefen emotionalisiert, und unter hohem publizistischem Aufwand als vorpommersche Identitätskrise in die regionale Öffentlichkeit gedrückt.

In Bezug auf die anfangs gestellten Arbeitsfragen ergeben sich folgende Befunde:

1. Wie funktioniert in einer Region die Abkopplung von nationalen und globalen Kommunikationsströmen, sodass sich ein regionales Identitätsproblem bilden kann? Bereits die Monopolstellung der regionalen „OstseeZeitung“ impliziert eine gewisse Abkopplung von der pluralistischen Meinungsvielfalt in Deutschland. Sie konnte und kann bis heute durch andere Medien wie Fernsehen oder Internet nicht ausgeglichen werden, denn diese sind erheblich stärker auf urbane, weitgehend regionsfremde Inhalte aufgerichtet. Während nach der Stadt-Land-Gliederung der EU 2018 im deutschen Durchschnitt nur 20,3\% der Bevölkerung in ländlichen Räumen wohnen, sind es in Mecklenburg-Vorpommern $44,9 \%$. Diese Räume wurden durch räumliche Zentralisierungsmaßnahmen der Landesadministration weiter marginalisiert. 1990 bis 2013 stand dem Bevölkerungsverlust in Höhe von $17 \%$ eine Reduzierung der Stadt- und Landkreise um $72 \%$ gegenüber. Die Negativprognosen der administrativen Gewalt zur Bevölkerungsentwicklung bewahrheiteten sich vorerst nicht. Seit 2013 weist die Bevölkerung des Landes ein verhaltenes Wachstum auf. „Jede Zerstörung einer demokratischen Struktur, einer demokratischen Institution wie die eines Landkreises oder eines demokratischen Unternehmens in der Peripherie stärkt undemokratische, außerparlamentarische, verfassungsfeindliche Kräfte“ (Klüter, 2018:29). Die demokratische Partizipationskette (s. o.) ist für die amtsangehörigen Gemeinden in den Landkreisen Mecklenburg-Vorpommerns gebrochen. Die kollektive Nichtbeachtung, die die Menschen 
in diesen Regionen erfahren, betrifft neben der Politik vor allem die städtisch geprägte Medienwirtschaft und pflanzt sich in große Teile der noch stärker urbanisierten Wissenschaft fort.

2. Wie wird ein regionales Problem zur Krise? Die Aufmerksamkeitsschranke für Probleme ländlicher oder peripherer Räume liegt inzwischen sehr hoch. Sie werden erst dann von den urban dominierten Medien wahrgenommen, wenn sie eine bestimmte Intensität überschritten haben.

Grundsätzlich ist das, was für das Unternehmen oder die Behörde oder die Institution eine Krise ist, für uns als Medium eher ein Skandal ... Skandale sind keine Naturereignisse, sondern werden lanciert und sie werden eben gelegentlich von den Medien auch aufgebauscht. Sie haben eine Dramaturgie. Medien haben im Prinzip drei Aufgaben. Sie wollen informieren, unterhalten und Service bieten. Im Fall des Skandals handelt es sich sicherlich zu einem gewissen Teil um Information, aber zu einem nicht unwesentlichen Teil auch um Unterhaltung des Publikums. Ein Skandal ist einfach auch spannender Lesestoff, ein Aufreger, die uralte Geschichte von Gut und Böse (Wewetzer, 2006:57).

Für die Greifswalder Lokalredaktion der OstseeZeitung als Monopol-Zeitung im nördlichen Vorpommern war es relativ einfach, die Rückbenennung der Universität als Skandal zu inszenieren. Möglicherweise hat sie nicht damit gerechnet, dass die neue Rechte dies als Anlass nehmen würde, sich und anderen ihre neu gewonnene Stärke, darunter auch die Organisationskraft des neuen Kreisverbands der AfD, zu zeigen und ihre Macht an dessen Standort, eben in Greifswald, zu demonstrieren. Davon, dass Demonstration und Gegendemonstration in Krawall und regionale Krise ausarteten, profitierten zunächst beide - Lokalredaktion und neue Rechte.

3. Inwieweit stützt ein derartiger (Krisen-)Regionalismus konservative und/oder rechtsgerichtete Tendenzen? Die Rückbenennungskrise bescherte der politischen Rechten ein regional vermarktbares Gesicht: Ernst Moritz Arndt. Sie reaktivierte die Mär vom knorrigen, aufrecht gegen das kosmopolitische Establishment kämpfenden, angeblich typisch pommerschen Charakter obwohl der reale Arndt eher dem Muster des Ost-WestMigranten entspricht, denn er verbrachte die meiste Zeit seines Lebens im Rheinland. Arndts Mythos war bereits in der Kaiserzeit, in der späten Weimarer Republik und während des Nationalsozialismus aufgebaut und gepflegt worden. Die Streichung Arndts aus dem Universitätsnamen tat dem neuen, eigentlich alten ArndtNarrativ wenig Abbruch. Der Innenminister gab höchstpersönlich die Deutung der Rückbenennungskrise für die politische Rechte vor: Der gute Arndt wurde zu einem Opfer der „Linksgrünen“ und kann nun posthum als rechtskonservativer Märtyrer verklärt werden (s.o.; Caffier, 2018).

Von den 36 Gemeinden Mecklenburg-Vorpommerns, in denen die AfD bei den Kreistagswahlen am 26. Mai 2019 zur stimmenstärksten Partei aufstieg, lagen 4 in der westlichen und 32 in der östlichen Landeshälfte. Dort waren es in Vorpommern-Rügen 3, auf der Mecklenburgischen Seenplatte 9 und in VorpommernGreifswald 20 Gemeinden. Dieser und andere Befunde bestätigen, dass die Stärke der politischen Rechten keineswegs nur von Peripherisierung, regionaler Armut oder ruralen Verstetigungstendenzen (vgl. Förtner et al., 2019) beeinflusst wird - denn dies beträfe ja auch viele Gemeinden in der westlichen Landeshälfte. Mindestens ebenso bedeutsam sind die politischen Wettbewerbsvorteile den demokratischen Parteien gegenüber, die der weitgehend autoritären AfD durch die drastische Kreisgebietsreform im Osten des Landes zugespielt wurden, und die Art und Weise, in der die politische Rechte lokal und regional entstandene Probleme und DemokratieDefizite zur eigenen Profilierung nutzt.

4. Gilt die im bayrischen Kontext aufgedeckte rechtsradikale Strategie „Wir erobern die Städte vom Land aus" (Becher, 2012:28) auch für Mecklenburg-Vorpommern? Der Aufbau einer Opposition gegen die Rückbenennung der Universität, die Durchführung von Demonstrationen und anderer Events dagegen war zunächst ein rein städtisches Projekt der rechtsgerichteten Gruppen. Dabei hat vielleicht unterstützend gewirkt, dass der 2013 gegründete Kreisverband die Aktivitäten „vom Lande“, also aus Ueckermünde, Pasewalk, Anklam und Wolgast, bündelte. Es ist schwer von der Hand zu weisen, dass es ohne die von der Schweriner Landesadministration verordnete Kreisgebietsreform einen derart zentralisierten, schlagkräftigen Kreisverband in Greifswald nicht geben würde. Die Landesadministration hat somit die organisatorische Hülle dafür geliefert, dass die Greifswalder AfD in der dargelegten Weise aus einem Nischendasein ins Zentrum gerückt wurde. Die lokale Stärke der AfD ist ein urbanes Phänomen, die sich mit einer Hochschulbenennung, also einem urbanen Thema, profilierte. Deutlich hat sie gezeigt, dass sie die rechte „Schmuddelecke“ der Hinterwäldler verlassen und sogar in einer Universitätsstadt Salonfähigkeit den Parteien der bürgerlichen Mitte gegenüber erlangen kann. Der Widerstand gegen die Rückbenennung der Universität geriet zu einer eindrucksvollen Machtdemonstration der neuen Rechten. Es wurde bereits darauf verwie- 
sen, dass Arndts Ideologie besonders für Kleinbürger attraktiv war. Heute sind es niedere und mittlere Angestellte und Beamte, die überproportional AfD wählen. Bei den Kommunalwahlen 2019 errang die AfD 40\%, die CDU jedoch nur $33 \%$ ihrer landesweiten Stimmen in Städten mit mehr als 10000 Einwohnern. Somit dürfte die AfD in Mecklenburg-Vorpommern bereits stärker auf die städtische Klientel orientiert sein als die CDU. Es ist zu erwarten, dass diese neue, urbanisierte AfD auch in anderen Städten nach dem Greifswalder Vorbild aktiv wird.

5. Kann die These „Gesellschaftliche Krisenphänomene ermöglichen rechtsextreme Rekrutierung" für Mecklenburg-Vorpommern bestätigt werden? Die Angehörigen der administrativen (vgl. Klüter, 2000b, 2009) und judikativen Gewalt sind einem besonderen Spannungsverhältnis ausgesetzt: Einerseits sollen sie Einrichtungen und Aktivitäten der Exekutiven schützen. Andererseits sind sie es, die dem konkreten oder indirekten Widerstand gegen räumliche Zentralisierungsmaßnahmen der Landesadministration, wie die Schließung von Schulen, Verwaltungen, Krankenhausabteilungen und kulturellen Einrichtungen, also der „Demokratie von unten“, ausgesetzt sind. Daher fordern viele Polizisten, Richter und Verwaltungsbeamte einen stärkeren Staat, engagieren sich offen oder verdeckt für die AfD und andere rechtsgerichtete Gruppen. Jedoch ist die offizielle Zahl der AfD-Mitglieder in den letzten zwei Jahren in Mecklenburg-Vorpommern kaum gestiegen, was nicht zuletzt durch die Abspaltung der „Bürger für Mecklenburg-Vorpommern“ bedingt ist. Die politisch rechte Szene konsolidiert sich jedoch nach den Verfassungsschutzberichten immer weiter. Der Zuzug von Aktiven und Sympathisanten aus anderen Bundesländern hält an.

6. Welche Aspekte regionaler Krisen sind dauerhaft wirksam, welche sind verallgemeinerungsfähig? Ähnlich wie nationale oder übernationale Krisen implizierte die Rückbenennungskrise zunächst eine Überforderung des eigentlich dafür zuständigen gesellschaftlichen Teilsystems - in diesem Fall der Wissenschaft - und der dafür zuständigen formalen Organisationen - hier der Universität und des Bildungsministeriums. Das Defizit an Entscheidung und Handlung ermöglichte es anderen formalen Organisationen - in diesem Fall der OstseeZeitung und den beiden Bürgerinitiativen - diese Lücke für sich zu nutzen. Im Vorfeld des anstehenden Bundestagswahlkampfs 2017 war keiner der neuen Akteure an einer Deeskalation interessiert.

Zur Deeskalation kam es erst, als die Universität mit einer neuen Satzung ihre Handlungsfähigkeit wiederherstellte, und in geteilter Verantwortlichkeit zwischen Hochschule und Ministerium die Initiative zur Rückbe- nennung übernommen wurde. Nun war es die Gegenseite, die mit der komplizierten juristischen Konstruktion und ihren weitreichenden Implikationen überfordert schien.

Im Anschluss an die Greifswalder Ereignisse kam es auch an anderen Orten zu Umbenennungsversuchen für Institutionen und Straßen, die nach Ernst Moritz Arndt benannt waren. Als Beispiele seien genannt:

- Die politische Rechte und die AfD nutzten ihre in Greifswald gesammelten Erfahrungen, um mit anderen politischen Gruppen die eigentlich anstehende Umbenennung der Ernst-Moritz-Arndt-Kaserne in Hagenow zu verhindern.

- Am 6. Mai 2019 beschloss die Ernst-Moritz-ArndtKirchengemeinde in Berlin-Zehlendorf, ihren Namen abzulegen. Allerdings ist der alte Name nach wie vor bis zum Beschluss über einen neuen gültig.

- Bizarr mutet das Vorgehen der Stadt Wien an: Am 4 Dezember 2018 erfuhr die dortige Arndtstraße im Unbehagen über die gesunkene Reputation ihres Namenspatrons eine „Ergänzungsbenennung“ mit dem Namen der Holocaust-Überlebenden Ilse Arndt (19132003). In https://www.geschichtewiki.wien.gv.at/ Arndtstra $\{$ Iss $\}$ e (letzter Zugriff: 11. Juni 2020) stehen jetzt beide Namen nebeneinander: der Rassist und Antisemit Ernst Moritz Arndt und die ehemalige KZ-gefangene Jüdin Ilse Arndt.

Als die Stadt Leipzig ihre Arndtstraße umbenennen wollte, bildete sich dort eine Bürgerinitiative zum Erhalt des alten Namens.

Die Greifswalder Rückbenennungskrise kristallisierte sich an der Zweckmäßigkeit der Nutzung einer regionalen historischen Persönlichkeit für heutige Marketing- und Präsentationsaufgaben. Derartige Persönlichkeiten findet man natürlich nicht nur in Vorpommern, sondern in allen Regionen. Insofern ist die Thematik tendenziell unerschöpflich - auch in städtischen Kontexten. Mit dem Wandel der gesellschaftlichen Zwecke und Traditionen ergeben sich immer neue Selektionsfilter für die regionale Geschichte. Die rechte Strategie, regionale „Helden“ in Beschlag zu nehmen und über unklare Felder der Erinnerungskultur die bürgerliche Mitte anzusprechen, ist somit nicht auf Ostdeutschland beschränkt. Erinnert sei an die Aktivitäten, die im Münsterland durch die Umbenennungsbestrebungen von Plätzen und Straßen ausgelöst wurden, die nach Karl Wagenfeld benannt worden waren. Auch die Auseinandersetzungen um das Agnes-MiegelDenkmal in Bad Nenndorf konnten von der politischen Rechten geschürt und für ihre Ziele ausgenutzt werden.

Die sich während der Krise abzeichnende plötzlich neugewonnene Stärke der politischen Rechten innerhalb von Greifswald ist aus überregionaler Sicht nichts Neues. „Wir 
haben als mehr oder weniger bürgerliche Stadt die Kreisfreiheit verloren und sind als politisches Zentrum der Rechten im Vogtlandkreis aufgewacht." So charakterisierte ein Politiker in Plauen die Situation seiner Stadt, die wie Greifswald nach der Kreisgebietsreform - in Sachsen bereits 2008/2009 - im Jahre 2013 Sitz eines großen AfD-Kreisverbands und anderer rechtsradikaler Strukturen wurde. Ähnliches geschah mit Görlitz und Zwickau. Die neuen Kreisverbände der AfD bündeln nicht nur die Aktivitäten der bis dahin stark lokal, also dezentral operierenden Rechten, sie entwickeln und stärken auch rechtsorientierte Gruppen in den Städten, in denen ihre Verbände residieren.

Die nach innen hierarchisch und auf Befehl-Gehorsam wie in Kasernen - ausgerichtete politische Rechte profitiert somit strukturell von der räumlichen Zentralisierung durch Kreisgebietsreformen, während die auf Partizipation, parlamentarische Diskussion und Wettbewerb angewiesenen demokratischen Parteien mit jedem aufgelösten Kreistag ein regionales Podium ihrer politischen Präsentationsmöglichkeiten verlieren. In Brandenburg und Thüringen bildeten sich daher 2016/2017 parteiübergreifende Initiativen, in der Kommunalpolitiker aller demokratischen Parteien - einschließlich der Regierungsparteien im Landtag - gegen die jeweilige Landesadministration und die von dort geplanten Kreisgebietsreformen protestierten. Sie konnten die dortigen Landtagsinnenausschüsse und Ministerpräsidenten schließlich überzeugen (vgl. Klüter, 2017).

Aus methodischer Sicht stellen sich dabei mehrere Fragen: Was darf Demokratie in ländlichen Räumen kosten? Bis zu welchem Grade lässt Demokratie als politisches System sich monetarisieren? Das neoliberale Modell einer um jeden Preis sparsamen Verwaltung muss schon per Sachzwang einen Kreistag mit seinen zeit- und finanzaufwändigen Entscheidungsstrukturen zum unnötigen Kostenfaktor erklären und trifft sich darin mit dem demokratieskeptischen Paternalismus, den Ernst Forsthoff und seine Schüler tief in die bundesdeutschen Verwaltungsgesetze eingepflanzt haben. Wenn „,regressive Moderne“ das impliziert, sollte man Oßenbrügge und anderen zustimmen.

Ähnlich wie bei anderen Krisen kam es bei dem Rückbenennungskonflikt zu außergewöhnlichen Maßnahmen, Aktivitäten und Lösungsversuchen. In Greifswald war es die nahezu reibungslose Zusammenarbeit zwischen Vertretern der CDU, der AfD und anderen rechtsorientierten Gruppen, die unter dem Deckmantel der Bürgerinitiative „Ernst Moritz Arndt bleibt" notdürftig kaschiert wurde. Sie bezog sich nicht nur auf die Inhalte, sondern auch auf die Diffamierungsinstrumente - wie beispielsweise die Prangerrede -, die von der CDU aus dem rechtsextremen Repertoire übernommen wurde. Die oben erwähnte Rückbenennungsgegnerin, die 2013 erste Vorsitzende des AfD-Kreisverbandes geworden war, wurde nach ihrem Ausscheiden aus der AfD von der CDU ebenfalls übernommen und durfte bei der Kommunalwahl am 2019 in Greifswald als Kandidatin antreten. Dies trug möglicherweise dazu bei, dass nicht die AfD, sondern

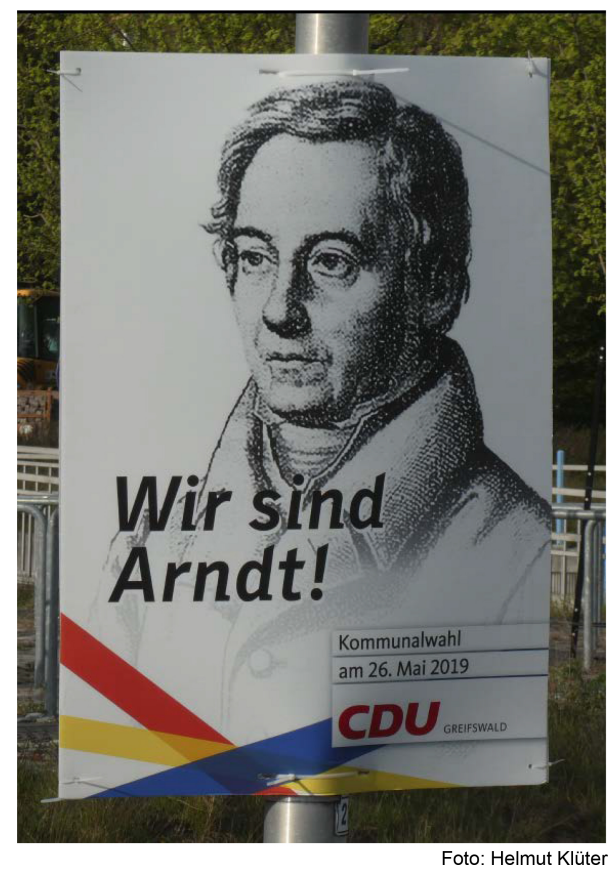

Abb. 5. Wahlplakat im Kommunalwahlkampf 2019 in Greifswald. Foto: Helmut Klüter.

die Stadt-CDU mit einer Arndt-Ikone in den Wahlkampf zog (vgl. Abb. 5).

Damit löste die lokale CDU für sich selbst eine Identitätskrise aus. Ihre Versuche, die AfD rechts zu überholen, wurden vom Wähler abgestraft. Ihr Stimmenanteil fiel von $30,7 \%$ im Jahre 2014 auf 20,1 \% 2019. Ihre Verluste in der Universitätsstadt übertrafen den Landesdurchschnitt erheblich.

Angesichts des enormen Drucks von außen und der zahlreichen Einschüchterungsversuche ist es bewundernswert, dass die Senatsmitglieder und das Rektorat die Rückbenennung beschlossen und umgesetzt haben. Positiv wurde von großen Teilen der Bevölkerung und von regionalen Unternehmen bewertet, dass die Universität die Namensfrage im zweiten Anlauf selbständig gelöst hat, ohne dass Eingriffe seitens des Ministeriums oder der Gerichte notwendig wurden.

Zwar wurde das Ergebnis der Rückbenennung überregional zur Kenntnis genommen, doch ihre teilweise dramatischen Umstände und Hintergründe wurden nicht dargestellt. Der eigentliche Skandal, die kaum verhüllte Zusammenarbeit der radikalen Rechten mit der bürgerlichen Mitte, wurde nicht zum Thema gemacht. Gerade dies wäre aber nötig gewesen, um zu zeigen, wie weit Rechtsradikalisierung in einigen Regionen fortgeschritten ist, durch welche Koalitionen sie weiter aufgewertet wird und wie stark sie durch räumliche Überzentralisierung staatlicher Einrichtungen begünstigt wird. Das Greifswalder Beispiel zeigt auch, was Stadt und Hochschule leisten müssen, um sich in einem solchen Um- 
feld erfolgreich zu behaupten, und was darüber hinaus nötig wäre, um Rechtsradikalismus wirksam zu bekämpfen.

Datenverfügbarkeit. Für diesen Artikel wurden nur Daten der öffentlichen Statistik genutzt.

Interessenkonflikt. Der Autor erklärt, dass kein Interessenkonflikt besteht.

Danksagung. Simon Runkel und Jonathan Everts danke ich für interessante Anregungen, den Gutachter_innen für spannende Hinweise, Benedikt Korf für Geduld und Herausgeberschaft sowie Janina Schulz für das aufmerksame Lektorat.

Begutachtung. This paper was edited by Benedikt Korf and reviewed by two anonymous referees.

\section{Literatur}

Arndt, E. M.: Katechismus für den Deutschen Kriegs- und Wehrmann, worin gelehret wird, wie ein christlicher Wehrmann seyn und mit Gott in den Streit gehen soll, online aufrufbar: https://download.digitale-sammlungen.de/pdf/ 1552315263bsb10263981.pdf (letzter Zugriff: 4. Oktober 2019), 1813.

Arndt, E. M.: Erinnerungen aus dem äußeren Leben, 3. Auflage, Weidmann, Leipzig, online aufrufbar: https://download. digitale-sammlungen.de/pdf/1477918110bsb10061433.pdf (letzter Zugriff: 3. Oktober 2016), 1842.

Arndt, E. M.: Pro populo Germanico, Reimer, Berlin, online aufrufbar: https://download.digitale-sammlungen.de/pdf/ 1490207310bsb10015226.pdf, (letzter Zugriff: 22. März 2017), 1854.

Ascher, S.: Die Germanomanie. Skizze zu einem Zeitgemälde, Achenwall, Berlin, online aufrufbar: https://haab-digital. klassik-stiftung.de/viewer/image/802732291/3/ (letzter Zugriff: 23. März 2018), 1815.

Bauer, P.: Die Namensdebatte der Greifswalder Universität 2016/2017 in der medialen Öffentlichkeit, Steinbecker Verlag, Greifswald, 2018.

Baumgartner, W., Buchholz, W., Buchstein, H., Gratz, M., Klüter, H., Lampe, R., Lutz, H., Stamm-Kuhlmann, T., Niendorf, M., Schumacher, E., Strauß, F., and Tenhaef, P.: Für die Universität Greifswald, Selbstverlag, Greifswald, online aufrufbar: http://xn--fr-die-universitt-greifswald-lnc81e.de/ wp-content/uploads/2017/10/EMAZ-web.pdf (letzter Zugriff: 7. März 2020), 2017.

Becher, M.: „Wir erobern die Städte vom Land aus“ - Rechtsextremismus in der Region, in: ASG - Ländlicher Raum, Heft 2, 28-30, online aufrufbar: https://www.asg-goe.de/pdf/LR0212. pdf (letzter Zugriff: 23. September 2018), 2012.

Behm, T.: Auf rechtem Kurs? Die AfD in MecklenburgVorpommern, in: Weiterdenken, 3, online aufrufbar: https: //library.fes.de/pdf-files/bueros/schwerin/11239.pdf (letzter Zugriff: 9. Januar 2020), 2015.

Caffier, L.: Ich bin enttäuscht über die Entscheidung des Senats, Facebook-Eintrag vom 17. Januar 2018, online aufrufbar: https: //www.facebook.com/caffier.lorenz/posts/1652159698183582 (letzter Zugriff: 12. November 2019), 2018.

Creuzberger, S., Mrotzek, F., und Niemann, M. (Hrsg.): Land im Umbruch. Mecklenburg-Vorpommern nach dem Ende der DDR. (= Diktatur und Demokratie im 20. Jahrhundert, Band 4), be.bra Wissenschaft, Berlin, 2018.

Eberle, H.: „Ein wertvolles Instrument“, Die Universität Greifswald im Nationalsozialismus, Böhlau, Köln, Weimar, Wien, 2015.

Erhart, W. and Koch, A. (Hrsg.): Ernst Moritz Arndt (1769-1860). Deutscher Nationalismus, Europa, transatlantische Perspektiven, Niemeyer, Tübingen, 2007.

Fina, S., Osterhage, F., Rönsch, J., Rusche, K., Siedentop, S., Zimmer-Hegmann, R., and Danielzyk, R.: Ungleiches Deutschland. Sozioökonomischer Disparitätenbericht 2019. Karten. Indikatoren und wissenschaftliche Handlungsempfehlungen für ein besseres Morgen, online aufrufbar: http://library.fes.de/pdf-files/ fes/15889.pdf (letzter Zugriff: 4. Februar 2020), 2019.

Förtner, M., Belina, B., and Naumann, M.: Stadt, Land, AfD, Zur Produktion des Ruralen im Prozess der Urbanisierung, Suburban, Z. Krit. Stadtforsch., 7, 23-44, 2019.

Grillparzer, F.: Sämtliche Werke, Ausgewählte Briefe, Gespräche, Berichte, in: Erster Band: Gedichte, Epigramme, Dramen I. Hanser, München, 1960.

Hesse, J. J.: Auswirkungen der Landkreisneuordnung. Abschlussbericht (2014/2015), Landtag Mecklenburg-Vorpommern, Drucksache 7/71, 18. November 2016.

Holm, L.-E.: Ernst-Moritz-Arndt-Universität behält Namen. Ministerin bestätigt AfD-Forderung, Presseerklärung der AfDMV vom 7. März 2017, online aufrufbar: https://www.afdmv.de/aktuelles/2017/03/ernst-moritz-arndt-universitaet-

behaelt-namen-ministerin (letzter Zugriff: 10. Oktober 2019), 2017.

Huntington, S. P.: The clash of civilizations. Deutsch: Kampf der Kulturen, Europaverlag, München, Wien, 1996.

Klüter, H.: Raum als Element sozialer Kommunikation, Giessener Geographische Schriften 60, Giessen, 1986.

Klüter, H.: Räumliche Aspekte von Transformationsproblemen aus systemtheoretischer Perspektive, Europa regional, 8, 35-51, online aufrufbar: https://www.ssoar.info/ssoar/handle/document/ 48272 (letzter Zugriff: 8. November 2019), 2000a.

Klüter, H.: Regionale Kommunikation in Politik und Wirtschaft, in: Informationen zur Raumentwicklung 27, Heft 9/10, 599-610, online aufrufbar: https://www.researchgate.net/publication/ 338864656_2000_Regionale_Kommunikation_in_Politik_und_ Wirtschaft (letzter Zugriff: 9. Januar 2020), 2000b.

Klüter, H.: Raum und Kompatibilität, in: Geographische Zeitschrift 90, Heft 3+4, 142-156, online aufrufbar: https://www.researchgate.net/publication/261776777_Raum_ und_Kompatibilitat (letzter Zugriff: 17. April 2020), 2002.

Klüter, H.: Demokratie und Verwaltung. Demokratische Prozesse und Verwaltungshandeln in der Regional- und Stadtentwicklung Mecklenburg-Vorpommerns, Greifswald, online aufrufbar: https://www.researchgate.net/publication/ 338645712_Demokratie_und_Verwaltung_-_Demokratische_ Prozesse_und_Verwaltungshandeln_in_der_Regional-_und_ 
Stadtentwicklung_Mecklenburg-Vorpommerns (letzter Zugriff: 17. April 2020), 2009.

Klüter, H.: Die Landwirtschaft Mecklenburg-Vorpommerns im Vergleich mit anderen Bundesländern, Greifswalder Geographische Arbeiten, 53, Greifswald 2016, online aufrufbar: https: //geo.uni-greifswald.de/fileadmin/uni-greifswald/fakultaet/ mnf/geowissenschaften/Institutsseiten/Seite_Schriftenreihen/ Greifswalder_Geographische_Arbeiten/GGA_53.pdf (letzter Zugriff: 8. Juni 2019), 2016.

Klüter, H.: Anmerkungen zum Gesetz zur Neugliederung der Landkreise und kreisfreien Städte im Land Brandenburg und zur Änderung anderer Gesetze nebst Anlagen. Vorgetragen auf der Anhörung des Landtags Brandenburg zum Neugliederungsgesetz am 20. Oktober 2017 im Gebäude des Landtags, Potsdam, online aufrufbar: https://www.researchgate.net/publication/ 334051432_2017-10-20_Landtagsanhorung_Brandenburg_ Anmerkungen_zum_Gesetz_zur_Neugliederung_der_ Landkreise_und_kreisfreien_Stadte_im_Land_Brandenburg (letzter Zugriff: 9. März 2020), 2017.

Klüter, H.: Folgen der Kreisgebietsreform und Entwicklungschancen für das östliche Mecklenburg-Vorpommern. Neubrandenburg, Greifswald, online aufrufbar: https: //www.researchgate.net/publication/334051208_Folgen_ der_Kreisgebietsreform_und_Entwicklungschancen_fur_ das_ostliche_Mecklenburg-Vorpommern (letzter Zugriff: 9. März 2020), 2018.

Klüter, H.: Flächen im ländlichen Raum MecklenburgVorpommerns, Wismar, online aufrufbar: https://www. researchgate.net/publication/334051455_Flachen_im_ landlichen_Raum_Mecklenburg-Vorpommerns (letzter Zugriff: 9. Januar 2020), 2019.

Kohr, L.: „Small is beautiful“ - ausgewählte Schriften, Deuticke, Wien, 1995.

Korf, B. und Schetter, C.: Krisenregion, in: Ortsregister. Ein Glossar zu Räumen der Gegenwart, Herausgeber: Marquardt, N. und Schreiber, V., transcript, Bielefeld, 148-153, 2012.

Leibert, T.: Europawahl 2019: Grüne Städte, schwarzes Land, blauer Osten?, in: Nationalatlas aktuell 14 (07.2019) 4, LeibnizInstitut für Länderkunde, Leipzig, online aufrufbar: http: //aktuell.nationalatlas.de/Europawahl_2019-4_07-2019-0-html/ (letzter Zugriff: 9. Januar 2020), 2019.

Leibert, T. and Haunstein, S.: Wahlverhalten macht zunehmende Differenzierung der Gesellschaft deutlich, Nationalatlas aktuell 12 (06.2018) 3, Leibniz-Institut für Länderkunde, online aufrufbar: http://aktuell.nationalatlas.de/Wahlgeographie.3_ 06-2018.0.html (letzter Zugriff: 9. Januar 2020), 2018.

Lippmann, W.: Public opinion. Harcourt, Brace, New York (Deutsch: Die öffentliche Meinung, Rütten und Loening, München 1964, Universitätsverlag, Bochum, 1990, Westend, Frankfurt/Main 2018), online aufrufbar: https: //ia600200.us.archive.org/22/items/publicopinion00lippgoog/ publicopinion00lippgoog.pdf (letzter Zugriff: 18. März 2019), 1922.

Luhmann, N.: Soziale Systeme, Grundriß einer allgemeinen Theorie, Suhrkamp, Frankfurt am Main, 1984.

Luhmann, N.: Die Gesellschaft der Gesellschaft, 2 Bde, Suhrkamp, Frankfurt am Main, 1997.
Merkel, W. (Hrsg.): Demokratie und Krise. Zum schwierigen Verhältnis von Theorie und Empirie, Springer, Wiesbaden, 2015.

Metapedia (Hrsg.): Ernst Moritz Arndt, online aufrufbar: https: //de.metapedia.org/wiki/Arndt,_Ernst_Moritz, letzter Zugriff: 11. Dezember 2019.

Morris, W.: News from Nowhere (Deutsch von Natalie Liebknecht und Clara Steinitz: Kunde von Nirgendwo. Mit einer Vorbemerkung von Wilhelm Liebknecht, Stuttgart, 1900, Nachdruck: Golkonda, Berlin 2013), 1890.

Müller, J.-W.: Was ist Populismus?, Suhrkamp, Berlin, 2016.

Münkler, H.: Partizipation vs. Wohlstand. Die westliche Demokratie in der Krise, Frankfurter Hefte, 12, 15-19, 2017.

Oßenbrügge, J.: „March for Sozialgeographie“? Rechtspopulismus als Zumutung und die regressive Moderne als Herausforderung der Humangeographie, Geogr. Helv., 73, 309-319, https://doi.org/10.5194/gh-73-309-2018, 2018.

Runkel, S. and Everts, J.: Geographien sozialer Krisen/Krisen sozialer Geographien, Geogr. Helv., 72, 475-482, https://doi.org/10.5194/gh-72-475-2017, 2017.

Rösel, F. and Sonnenburg, J.: Politisch abgehängt? Kreisgebietsreform und AfD-Wahlergebnis in Mecklenburg-Vorpommern, Ifo Dresden berichtet, 6, 6-13, 2016.

Schmitthenner, H.: Lebensräume im Kampf der Kulturen, 1. Auflage 1938, neu aufgelegt: Quelle \& Meyer, Heidelberg, 1951.

Siebenpfeiffer, P. J.: Rede von Dr. Philipp Jakob Siebenpfeiffer auf dem Hambacher Fest, online aufrufbar: http://www.demokratiegeschichte.eu/fileadmin/user_upload/ Material/Rede_Siebenpfeiffer.pdf (letzter Zugriff: 8. November 2019), 1832.

Sloterdijk, P.: Die schrecklichen Kinder der Neuzeit, Suhrkamp, Berlin, 2015.

Universität Greifswald (Hrsg.): Leitbild der Universität, beschlossen am 10. Oktober 2012, online aufrufbar: https://www. uni-greifswald.de/fileadmin/uni-greifswald/1_Universitaet/1. 1_Information/1.1.1_Ueber_uns/Leitbild/Leitbild.pdf (letzter Zugriff: 26. März 2019), 2012.

van den Daele, W.: Krise - Annäherung an ein Phänomen. Braucht die Gesellschaft ihre Krisen?, in: Was kostet eine Krise? Fakten, Erfahrungen, Handlungsmöglichkeiten, Herausgeber: Bundesinstitut für Risikobewertung, Berlin, 35-37, online aufrufbar: https://mobil.bfr.bund.de/cm/350/was_kostet_eine_krise_ fakten_erfahrungen_handlungsmoeglichkeiten_tagungsband.pdf (letzter Zugriff: 9. März 2020), 2006.

Verfassungsschutzbericht 2018 Mecklenburg-Vorpommern: herausgegeben vom Ministerium für Inneres und Europa Mecklenburg-Vorpommern, Schwerin, online aufrufbar: http://www.verfassungsschutz-mv.de/publikationen/, letzter Zugriff: 8. November 2019.

Vogt, H.: Nationalismus gestern und heute. Texte und Dokumente, Leske, Opladen, 1967.

Wewetzer, H.: Suchen Medien ihre Krisen?, in: Was kostet eine Krise? Fakten, Erfahrungen, Handlungsmöglichkeiten. Herausgeber: Bundesinstitut für Risikobewertung, Berlin, 57-60, online aufrufbar: https://mobil.bfr.bund.de/cm/350/was_kostet_ eine_krise_fakten_erfahrungen_handlungsmoeglichkeiten_ tagungsband.pdf (letzter Zugriff: 9. März 2020), 2006. 